\title{
Pacific
}

Journal of

Mathematics

\section{ON THE POSTULATION OF 0-DIMENSIONAL SUBSCHEMES ON A SMOOTH QUADRIC}

Salvatore Giuffrida, Renato Maggioni ANd Alfio RAgusa 


\title{
ON THE POSTULATION OF 0-DIMENSIONAL SUBSCHEMES ON A SMOOTH QUADRIC
}

\author{
S. Giuffrida, R. Maggioni, ANd A. Ragusa
}

\begin{abstract}
If $X$ is a 0-dimensional subscheme of a smooth quadric $Q \cong$ $\mathbf{P}^{1} \times \mathbf{P}^{1}$ we investigate the behaviour of $X$ with respect to the linear systems of divisors of any degree $(a, b)$. This leads to the construction of a matrix of integers which plays the role of a Hilbert function of $X$; we study numerical properties of this matrix and their connection with the geometry of $X$. Further we relate the graded Betti numbers of a minimal free resolution of $X$ on $Q$ with that matrix, and give a complete description of the arithmetically Cohen-Macaulay 0-dimensional subschemes of $Q$.
\end{abstract}

Introduction. In the last few years the interest about 0-dimensional subschemes of $\mathbf{P}^{n}$ has greatly grown, so many recent papers concern a deep investigation into the Hilbert function, free resolution, Betti numbers, and defining equations for such subschemes. On the other hand there has been a good deal of work on two codimensional subschemes of $\mathbf{P}^{n}$; hence, points of $\mathbf{P}^{2}$, which have both conditions, have been intensively studied. The interest on points of $\mathbf{P}^{2}$ comes, also, because geometric properties of a variety can sometimes be given in terms of its generic hyperplane section; so, for studying curves of $\mathbf{P}^{3}$, one needs properties of 0 -dimensional subschemes of $\mathbf{P}^{2}$. A complete list of papers on these topics seems impossible to do; so we insert in the references just a few of them, which are more familiar to us.

It seems natural to generalize this situation from one side studying 0 -dimensional subschemes of any variety and in particular of surfaces, on the other side working on sections of varieties done by hypersurfaces of degree bigger than one. Therefore, a first step in this direction is to investigate 0 -dimensional subschemes of a quadric $\left(\mathbf{P}^{1} \times \mathbf{P}^{1}\right)$ with special regard to their behaviour with respect to the divisors of the quadric itself.

When one embeds the quadric $Q$ in $\mathbf{P}^{3}$, any subscheme $X$ of $Q$ becomes a subscheme of $\mathbf{P}^{3}$; in that case one can relate properties of $X$ as a subscheme of $Q$ with those as a subscheme of $\mathbf{P}^{3}$.

Of course, studying subschemes of $Q$, the geometry of the surface $Q$ plays a big role; in particular, the cohomology groups of $Q$ play an 
important part; but, unfortunately, they do not vanish as the analogues on $\mathbf{P}^{n}$ do. This is one reason why subschemes of $Q$ with maximal codimension need not be arithmetically Cohen-Macaulay.

A very naive question arises at this point: given a set of points $X$ on a smooth quadric $Q$, how to compute its "Hilbert function" on $Q$, i.e. the number of conditions that $X$ imposes to the linear systems of curves on $Q$. Taking into account that $\operatorname{Pic} Q \cong \mathbf{Z} \oplus \mathbf{Z}$, one notices that the Hilbert function of $X$ takes the shape of a matrix: that is why we will call the postulation of $X$ "the Hilbert matrix". This kind of matter seems to be completely unexplored: as far as we tried, we could find no literature on it. Therefore, the results in this paper represent just a starting step in this field.

This point of view leads to quite surprising results: two points could be non-collinear on $Q$, since there are "too few lines" on it; moreover these points give the easiest example of a non-arithmetically CohenMacaulay 0-dimensional subscheme of $Q$. It comes out clearly how important it is to define the context of our investigation, and to use a proper nomenclature: this is the subject of the first section.

In $\S 2$ we investigate the structure of the Hilbert matrix of a 0 dimensional subscheme $X$ of $Q$, with special regard to the distribution of the points of $X$ on the lines of the two rulings.

The minimal free resolution of the ideal sheaf $\mathscr{I}_{X} \subset \mathscr{O}_{Q}$ of $X$, the relationships between the Hilbert matrix and the cohomology groups of $\mathscr{J}_{X}$ are the main ingredients of $\S 3$.

In the final section the arithmetically Cohen-Macaulay 0-dimensional subschemes of $Q$ are characterized in terms of their Hilbert matrix. Moreover, a complete description of their minimal free resolution is given.

For the definitions and the results which are not explicitly given, we refer to Hartshorne's book [H].

1. Notation and preliminaries. Let $\mathbf{P}^{1}=\mathbf{P}_{k}^{1} \quad(k$ an algebraically closed field), let $Q=\mathbf{P}^{1} \times \mathbf{P}^{1}$ be a quadric and let $\mathscr{O}_{Q}$ be its structure sheaf. If $D \subset Q$ is any divisor of type $(a, b)$ we denote by $\mathscr{O}_{Q}(a, b)$ the associated sheaf and, for any sheaf $\mathscr{F}$ on $Q$, we set $\mathscr{F}(a, b)=$ $\mathscr{F} \otimes \mathscr{O}_{Q}(a, b)$. We also use the following notation:

$$
H^{i}(a, b)=H^{i}\left(Q, \mathscr{O}_{Q}(a, b)\right), \quad h^{i}(a, b)=\operatorname{dim}_{k} H^{i}(a, b)
$$

and, for any sheaf $\mathscr{F}$ on $Q$

$$
\begin{aligned}
H^{i}(\mathscr{F}(a, b)) & =H^{i}(Q, \mathscr{F}(a, b)), \\
h^{i}(\mathscr{F}(a, b)) & =\operatorname{dim}_{k} H^{i}(\mathscr{F}(a, b)) .
\end{aligned}
$$


Let us consider

$$
S=H_{*}^{0}(a, b)=\bigoplus_{\substack{a \geq 0 \\ b \geq 0}} H^{0}(a, b)
$$

$S$ is in a natural way a $k$-algebra using product of sections. It is easy to check that $S$ is generated, as a $k$-algebra, by $H^{0}(1,0)$ and $H^{0}(0,1)$ (both vector spaces of dimension 2) since for every $a, b \geq 0$ the map

$$
H^{0}(a, b) \otimes H^{0}(1,0) \otimes H^{0}(0,1) \rightarrow H^{0}(a+1, b+1)
$$

given by the product is surjective (see Lemma 2.3 for a generalization).

$S$ is a bi-graded $k$-algebra taking $H^{0}(a, b)=S_{(a, b)}$ as the homogeneous component of degree $(a, b)$. When $s \in H^{0}(a, b)$, its zero locus $(s)_{0}$ will be called a curve of type $(a, b)$; in particular $L=(l)_{0}$ and $L^{\prime}=\left(l^{\prime}\right)_{0}$, with $l \in H^{0}(1,0)$ and $l^{\prime} \in H^{0}(0,1)$ will be mentioned as lines of type $(1,0)$ or $(1,0)$-lines, and lines of type $(0,1)$ or $(0,1)$-lines respectively. When no confusion can arise we will not distinguish between curves and their defining forms.

Let $u, u^{\prime}$ and $v, v^{\prime}$ be bases for $H^{0}(1,0)$ and $H^{0}(0,1)$; then we have a bi-graded ring isomorphism

$$
S \cong k\left[u, u^{\prime}\right] \otimes k\left[v, v^{\prime}\right]
$$

We use the above isomorphism to identify elements of $S$ and elements of $k\left[u, u^{\prime}\right] \otimes k\left[v, v^{\prime}\right]$. We deal only with bihomogeneous ideals of $S$, i.e. ideals generated by elements which are homogeneous both with respect to $u, u^{\prime}$ and $v, v^{\prime}$. From now on we will call them homogeneous ideals for short.

Consider the following subrings of $S: A=\bigoplus_{n \geq 0} H^{0}(0, n), B=$ $\bigoplus_{m \geq 0} H^{0}(m, 0)$; for a fixed $m \geq 0 \quad S_{(m,-)}=\bigoplus_{n \geq 0} H^{0}(m, n)$ inherits an $A$-module structure from $S$ and similarly $S_{(-, n)}=$ $\bigoplus_{m \geq 0} H^{0}(m, n)$ as $B$-module.

When $Q$ is embedded in $\mathbf{P}^{3}$ by the Segre embedding, the coordinate ring of $Q$ is $\bigoplus_{n \geq 0} H^{0}(n, n)$.

For the reader's convenience we recall the dimensions of the cohomology groups of $\mathscr{O}_{Q}(a, b)$ : 


$$
\begin{aligned}
& h^{0}(a, b)= \begin{cases}(a+1)(b+1) & \text { for } a, b \geq 0, \\
0 & \text { otherwise } ;\end{cases} \\
& h^{1}(a, b)= \begin{cases}-(a+1)(b+1) & \text { for } a \leq-2 \text { and } b \geq 0 \\
0 & \text { or } a \geq 0 \text { and } b \leq-2,\end{cases} \\
& h^{2}(a, b)= \begin{cases}(a+1)(b+1) & \text { otherwise } \\
0 & \text { otherwise }\end{cases}
\end{aligned}
$$

$h^{0}(a, b)$ is well known; $h^{2}(a, b)$ is obtained by Serre's duality; $h^{1}(a, b)$ can be computed by using the Riemann-Roch Theorem for surfaces. Note that for any divisor $D \subset Q$ (effective or not) of type $(a, b)$ the Euler characteristic of $\mathscr{O}_{Q}(a, b)$ is

$$
\chi\left(\mathscr{O}_{Q}(a, b)\right)=(a+1)(b+1)
$$

since only one among $H^{i}(a, b)(i=0,1,2)$ can be different from zero, that is $\mathscr{O}_{Q}$ has natural cohomology.

Let $P$ be any point on $Q$, i.e. the zero locus of an ideal $\mathfrak{p}=$ $\left(l\left(u, u^{\prime}\right) \otimes 1,1 \otimes l^{\prime}\left(v, v^{\prime}\right)\right)$ where $l$ and $l^{\prime}$ are linear forms; the element $\left(a, a^{\prime} ; b, b^{\prime}\right) \in k^{2} \times k^{2}$, homogeneous in $a, a^{\prime}$ and $b, b^{\prime}$, with $l\left(a, a^{\prime}\right)=0$ and $l^{\prime}\left(b, b^{\prime}\right)=0$ gives the coordinates of $P$ as subvariety of $Q$, with respect to the chosen basis.

Consider the following ideals of $S: \mathfrak{u}=\left(u \otimes 1, u^{\prime} \otimes 1\right), \mathfrak{v}=$ $\left(1 \otimes v, 1 \otimes v^{\prime}\right)$; their zero locus is trivially empty. An ideal $\mathfrak{a} \subset S$ is said to be irrelevant when it contains either a power of $\mathfrak{u}$ or a power of $\mathfrak{v}$. In the set of non-irrelevant homogeneous ideals of $S$ the maximal elements are the ideals of points, i.e. generated by $l\left(u, u^{\prime}\right) \otimes 1$, $1 \otimes l^{\prime}\left(v, v^{\prime}\right)$, where $l$ and $l^{\prime}$ are linear forms; this is seen looking at the restrictions of these ideals to the rings $k\left[u, u^{\prime}\right], k\left[v, v^{\prime}\right]$ and noting that such rings have principal non-irrelevant ideals. As a consequence one gets that an ideal $\mathfrak{a} \subset S$ is irrelevant iff $Z(\mathfrak{a})=\varnothing$. For any homogeneous ideal $\mathfrak{a} \subset S$ we define the saturation sat $\mathfrak{a}$ of $\mathfrak{a}$ to be

$$
\text { sat } \mathfrak{a}=\left\{f \in S \mid f \mathfrak{u}^{t} \subset \mathfrak{a} \text { for some } t\right\}+\left\{f \in S \mid f \mathfrak{v}^{t^{\prime}} \subset \mathfrak{a} \text { for some } t^{\prime}\right\} .
$$

By standard techniques one shows that Hilbert's Nullstellensatz holds in $S$ :

THEOREM 1.1. Let $\mathfrak{a} \subset S$ be a homogeneous saturated ideal and $f \in S$ a homogeneous element. If $Z(f) \supseteq Z(\mathfrak{a})$ then $f \in \sqrt{\mathfrak{a}}$. 
The next theorem gives basic information about the generators for a saturated ideal of height 2 of $S$.

THeOREM 1.2. Let $\mathfrak{a} \subset S$ be a saturated ideal of height 2. Then any minimal set of generators of a contains just one element of degree $(0, n)$ for some $n$ and just one element of degree $(m, 0)$ for some $m$.

Proof. Since $\mathfrak{a}$ is saturated of height 2 , then it is pure, so there exists an $S$-sequence $f, g$ in $\mathfrak{a}$. Consider the resultants $R_{1}\left(u^{\prime} \otimes 1\right)$ and $R_{2}(u \otimes 1)$ of $f$ and $g$ with respect to $u \otimes 1$ and $u^{\prime} \otimes 1$; these are elements of $\mathfrak{a}$ of the following type: $R_{1}=u^{\prime t} \otimes h^{\prime}\left(v, v^{\prime}\right), R_{2}=$ $u^{t} \otimes h\left(v, v^{\prime}\right)$ where $h$ and $h^{\prime}$ are forms with the same degree. Observe that $h\left(v, v^{\prime}\right)=h^{\prime}\left(v, v^{\prime}\right)$ : indeed they are resultants of $f$ and $g$ regarded as homogeneous polynomials in $u \otimes 1$ and $u^{\prime} \otimes 1$, and $f, g$ have no common components. Since $\mathfrak{a}$ is saturated $1 \otimes h^{\prime}\left(v, v^{\prime}\right) \in$ $\mathfrak{a}$. Similarly one proves that in $\mathfrak{a}$ there exists an element of degree $(m, 0)$. Uniqueness follows since the graded rings $k\left[u, u^{\prime}\right], k\left[v, v^{\prime}\right]$ have principal homogeneous ideals.

REMARK 1.3. As a consequence of the above theorem, a saturated ideal of $S$ of height 2 is a complete intersection iff it is generated by 2 elements of type $h\left(u, u^{\prime}\right) \otimes 1,1 \otimes h^{\prime}\left(v, v^{\prime}\right)$, where $h$ and $h^{\prime}$ are any forms. From now on we shall mean by complete intersection on $Q$ (c.i. for short) a subscheme whose saturated ideal has just 2 generators.

2. 0-dimensional subschemes of $Q$. Let $X \subset Q$ be a 0 -dimensional subscheme, i.e. a subscheme associated to a saturated ideal in $S$ of height 2 . In this paper we shall for simplicity concentrate on the case when $X$ consists of distinct points, but the results carry over to the general situation.

We can associate to any 0-dimensional subscheme $X$ of $Q$ the bigraded $S$-algebra $S(X)=S / I(X)$, where $I(X)$ is the homogeneous saturated ideal of $X$ in $S$. On the analogy of Hilbert function for graded modules, we can define the function

$$
M_{X}: \mathbf{Z} \times \mathbf{Z} \rightarrow \mathbf{N}
$$

by

$$
M_{X}(i, j)=\operatorname{dim}_{k}(S(X))_{(i, j)}=\operatorname{dim}_{k}(S)_{(i, j)}-\operatorname{dim}_{k}(I(X))_{(i, j)}
$$

where for every bi-graded $S$-module $N$ we denote by $(N)_{(i, j)}$ the component of degree $(i, j)$. If $\mathscr{I}_{X}$ is the ideal sheaf of $X$ in $Q$, we 
also have

$$
M_{X}(i, j)=h^{0}(i, j)-h^{0}\left(\mathscr{I}_{X}(i, j)\right) .
$$

The function $M_{X}$ produces a matrix with integer entries, $M_{X}=$ $\left(M_{X}(i, j)\right)$, which will be called the Hilbert matrix of $X$. Note that $M_{X}(i, j)=0$ for $i<0$ or $j<0$; so, from now on we restrict ourselves to the range $i \geq 0, j \geq 0$. When no confusion can arise we will use the notation $M_{X}=\left(m_{i j}\right)$ (warning: despite the name there is no relation between this matrix and the Hilbert-Burch matrix; but we will use this terminology since it seems the most natural).

From the defining exact sequence

$$
0 \rightarrow \mathscr{I}_{X} \rightarrow \mathscr{O}_{Q} \rightarrow \mathscr{O}_{X} \rightarrow 0
$$

taking cohomology we have:

$$
\begin{aligned}
h^{1}\left(\mathscr{I}_{X}(i, j)\right) & =h^{0}\left(\mathscr{I}_{X}(i, j)\right)-h^{0}(i, j)+h^{0}\left(\mathscr{O}_{X}(i, j)\right) \\
& =\operatorname{deg} X-m_{i j} \text { for } i, j \geq 0, \\
h^{2}\left(\mathscr{I}_{X}(i, j)\right) & =0 \text { for } i, j \geq 0,
\end{aligned}
$$

since $h^{2}\left(\mathscr{O}_{X}(i, j)\right)=0$ and in that range $H^{1}(i, j)=H^{2}(i, j)=0$.

It will be useful in the sequel to consider in $\mathbf{Z} \times \mathbf{Z}$ the partial ordering induced by the usual one on $Z$; we will denote it by " $\leq$ ".

REMARK 2.1. When one thinks of $Q$ as a subvariety of $\mathbf{P}^{3}$ by the Segre embedding, $X$ becomes a subscheme of $\mathbf{P}^{3}$. In this case, if $H F(X,-)$ is the Hilbert function of $X$ in $\mathbf{P}^{3}$, one has

$$
H F(X, i)=m_{i i} \text { for } i \geq 0 \text {. }
$$

This easily follows taking cohomology of the defining exact sequence of $Q$ in $\mathbf{P}^{3}$ and of the exact sequence

$$
0 \rightarrow \mathscr{I}_{Q} \rightarrow \mathscr{I}_{X}^{\prime} \rightarrow \mathscr{I}_{X} \rightarrow 0
$$

where $\mathscr{I}_{Q}$ and $\mathscr{I}_{X}^{\prime}$ are the ideal sheaves of $Q$ and $X$ in $\mathbf{P}^{3}$.

Let $M=\left(m_{i j}\right)$ be a matrix, with $i, j \in \mathbf{Z}$; we will use the following notation: we set

$$
\Delta^{R} M=\left(a_{i j}\right), \quad \Delta^{C} M=\left(b_{i j}\right)
$$

for the matrices of differences by rows and by columns of $M$, respectively. Thus we have $a_{i j}=m_{i j}-m_{i j-1}, b_{i j}=m_{i j}-m_{i-1 j}$. It is easy to check that $\Delta^{R}\left(\Delta^{C} M\right)=\Delta^{C}\left(\Delta^{R} M\right)$; this matrix will be denoted by $\Delta M=\left(c_{i j}\right)$ and referred to as the first difference matrix of $M$. The second difference matrix of $M$ is $\Delta^{2} M=\Delta(\Delta M)=\left(d_{i j}\right)$. 
Since for every $(h, k)$ one has $c_{h k}=m_{h k}+m_{h-1 k-1}-m_{h k-1}-$ $m_{h-1 k}$, when $M=M_{X}$ is the Hilbert matrix of a subscheme $X$ of $Q$ one sees that

$$
m_{i j}=\sum_{\substack{h \leq i \\ k \leq j}} c_{h k} \text { and } \quad c_{i j}=\sum_{\substack{h \leq i \\ k \leq j}} d_{h k} .
$$

Definition 2.2. Let $M^{\prime}=\left(m_{i j}^{\prime}\right)$ be a matrix such that $m_{i j}^{\prime}=0$ for $i<0$ or $j<0$. We say that $M^{\prime}$ is admissible when its first difference $\Delta M^{\prime}=\left(c_{i j}^{\prime}\right)$ satisfies the following conditions:

(1) $c_{i j}^{\prime} \leq 1$ and $c_{i j}^{\prime}=0$ for $i \gg 0$ or $j \gg 0$;

(2) if $c_{i j}^{\prime} \leq 0$ then $c_{r s}^{\prime} \leq 0$ for any $(r, s) \geq(i, j)$;

(3) for every $(i, j) \quad 0 \leq \sum_{t=0}^{j} c_{i t}^{\prime} \leq \sum_{t=0}^{j} c_{i-1 t}^{\prime}$, and $0 \leq \sum_{t=0}^{i} c_{t j}^{\prime} \leq \sum_{t=0}^{j} c_{t j-1}^{\prime}$.

When $M^{\prime}$ is an admissible matrix the non-zero part of $\Delta M^{\prime}$ is contained in a rectangle with opposite vertices $(0,0),(a, b)$ and the elements of the first row (resp. of the first column) are:

$$
\begin{gathered}
c_{0 j}^{\prime}=1 \text { if } j \leq b, \quad \text { and } c_{0 j}^{\prime}=0 \text { if } j>b \\
\text { (resp. } \left.c_{i 0}^{\prime}=1 \text { if } i \leq a, \text { and } c_{i 0}^{\prime}=0 \text { if } i>a\right) .
\end{gathered}
$$

In this case we say $M^{\prime}$, or $\Delta M^{\prime}$, to be of size $(a, b)$.

We will show that the Hilbert matrix of a 0-dimensional subscheme of $Q$ is admissible (see Propositions 2.5 and 2.7).

Lemma 2.3. Let $X \subset Q$ be a 0-dimensional subscheme. For the cup-product morphisms

$$
\begin{aligned}
\varphi_{i}: H^{0}\left(\mathscr{I}_{X}(i, j)\right) \otimes H^{0}(1,0) & \rightarrow H^{0}\left(\mathscr{I}_{X}(i+1, j)\right), \\
\psi_{j}: H^{0}\left(\mathscr{I}_{X}(i, j)\right) \otimes H^{0}(0,1) & \rightarrow H^{0}\left(\mathscr{I}_{X}(i, j+1)\right),
\end{aligned}
$$

we have:

$$
\begin{aligned}
\operatorname{dim}_{k} \operatorname{Im} \varphi_{i} & =2 h^{0}\left(\mathscr{I}_{X}(i, j)\right)-h^{0}\left(\mathscr{I}_{X}(i-1, j)\right), \\
\operatorname{dim}_{k} \operatorname{Im} \psi_{j} & =2 h^{0}\left(\mathscr{I}_{X}(i, j)\right)-h^{0}\left(\mathscr{I}_{X}(i, j-1)\right) .
\end{aligned}
$$

Proof. Let $s_{1}, s_{2}, \ldots, s_{r}$ be a basis of $H^{0}\left(\mathscr{J}_{X}(i-1, j)\right)$, where $r=$ $h^{0}\left(\mathscr{I}_{X}(i-1, j)\right)$, and let $u, u^{\prime}$ be a basis of $H^{0}(1,0)$ not vanishing at any point of $X$. Consider the following basis for $H^{0}\left(\mathscr{J}_{X}(i, j)\right)$ :

$$
s_{1} u, s_{2} u, \ldots, s_{r} u, s_{r+1}, \ldots, s_{n}
$$


where $n=h^{0}\left(\mathscr{J}_{X}(i, j)\right)$; notice that no element in the vector subspace spanned by $s_{r+1}, \ldots, s_{n}$ can contain $u$ as a component. Now, a standard computation shows that (see [GMa], Lemma 3.4)

$$
s_{1} u^{2}, s_{2} u^{2}, \ldots, s_{r} u^{2}, s_{r+1} u, \ldots, s_{n} u, s_{r+1} u^{\prime}, \ldots, s_{n} u^{\prime}
$$

is a basis for $\operatorname{Im} \varphi_{i}$. This proves the first part; the second part follows similarly.

REMARK 2.4. Observe that, for every $i \geq 0, \bigoplus_{j \geq 0} H^{0}\left(\mathscr{J}_{X}(i, j)\right)$ is a torsion-free $A$-module; since $A$ is a domain with principal homogeneous non-irrelevant ideals, this $A$-module is free (cf., e.g., [AF] Cap. II, §8). In particular, $S_{(i,-)}$ is $A$-free for every $i \geq 0$.

The same is true for $\bigoplus_{i \geq 0} H^{0}\left(\mathscr{I}_{X}(i, j)\right)$ and for $S_{(-, j)}$ as $B$ modules for every $j \geq 0$.

Proposition 2.5. Let $X \subset Q$ be a 0-dimensional subscheme, and $M_{X}=\left(m_{i j}\right)$ its Hilbert matrix. Then, the matrix $\Delta^{R} M_{X}$ (resp. $\left.\Delta^{C} M_{X}\right)$ is non-increasing by rows (resp. by columns), i.e. for every $(i, j) \geq$ $(0,0) a_{i j} \geq a_{i j+1}$ (resp. $\left.b_{i j} \geq b_{i+1 j}\right)$. Moreover $a_{i j}=0$ for $j \gg 0$ (resp. $b_{i j}=0$ for $i \gg 0$ ).

Proof. It is enough to prove the theorem for $\Delta^{R} M_{X}$. For simplicity we set $h_{i j}=h^{0}\left(\mathscr{J}_{X}(i, j)\right)$, so by Lemma 2.3 we have $h_{i j+1} \geq 2 h_{i j}-$ $h_{i j-1}$. Using $m_{r s}=(r+1)(s+1)-h_{r s}$ we get

$$
2 m_{i j}-m_{i j-1} \geq m_{i j+1}
$$

from which we obtain our result $a_{i j} \geq a_{i j+1}$ for every $(i, j) \geq(0,0)$.

For the second part we know that $m_{i i}=H F(X, i)=\operatorname{deg} X$ for $i \gg 0$; since in any case $m_{i j} \leq \operatorname{deg} X$, the conclusion follows using the first part.

REMARK 2.6. Let $i \geq 0$ be a fixed integer, and set

$$
q_{i}=\min \left\{j \mid h_{i j}>0\right\}
$$

where, as before, $h_{i j}=h^{0}\left(\mathscr{J}_{X}(i, j)\right)$. For every $j \geq q_{i}$ we set $\alpha_{i j}=$ $h_{i j}-\operatorname{dim}_{k} \operatorname{Im} \psi_{j-1}$ (see Lemma 2.3 for notation): note that $\alpha_{i j}$ is the number of minimal generators of degree $(i, j)$ for the $A$-module

$$
H_{*}^{0}\left(\mathscr{I}_{X}(i,-)\right)=\bigoplus_{j \geq 0} H^{0}\left(\mathscr{I}_{X}(i, j)\right)
$$


Applying Lemma 2.3 we have:

$$
\begin{aligned}
& \alpha_{i q_{i}}=(i+1)\left(q_{i}+1\right)-m_{i q_{i}} \\
& \alpha_{i q_{i}+1}=(i+1)\left(q_{i}+2\right)-m_{i q_{i}+1}-2 \alpha_{i q_{i}} \text {, } \\
& \alpha_{i q_{i}+2}=(i+1)\left(q_{i}+3\right)-m_{i q_{l}+2}-2 \alpha_{i q_{l}+1}-3 \alpha_{i q_{i}} \text {, } \\
& \alpha_{i j}=(i+1)(j+1)-m_{i j}-2 \alpha_{i j-1}-3 \alpha_{i j-2}-\cdots-\left(j+1-q_{i}\right) \alpha_{i q_{i}},
\end{aligned}
$$

from which we get

$$
h_{i j}=\alpha_{i j}+2 \alpha_{i j-1}+3 \alpha_{i j-2}+\cdots+\left(j+1-q_{i}\right) \alpha_{i q_{i}} .
$$

A simple computation shows

$$
a_{i j}=(i+1)(j+1)-h_{i j}-\left[(i+1) j-h_{i j-1}\right]=i+1-\sum_{t=q_{i}}^{j} \alpha_{i t} .
$$

This equality, since $a_{i j}=0$ for $j \gg 0$, shows that the $A$-free module $H_{*}^{0}\left(\mathscr{J}_{X}(i,-)\right)$ has $i+1$ generators. Of course the same happens for the $B$-free module $H_{*}^{0}\left(\mathscr{J}_{X}(-, j)\right)$.

Proposition 2.7. Let $X \subset Q$ be a 0-dimensional subscheme, and $M_{X}=\left(m_{i j}\right)$ its Hilbert matrix. Then for $\Delta M_{X}=\left(c_{i j}\right)$ we have:

(i) if $c_{i j} \leq 0$ then $c_{r s} \leq 0$ for every $(r, s) \geq(i, j)$;

(ii) if $c_{i j}>0$ then $c_{i j}=1$.

Proof. To prove (i) it is enough, for symmetry, to prove that if $c_{i j} \leq 0$ then $c_{r j} \leq 0$ for every $r \geq i$. Let us consider the following piece of the matrix $M_{X}$

$$
\begin{array}{ll}
m_{i-1 j-1} & m_{i-1 j} \\
m_{i j-1} & m_{i j} \\
m_{i+1 j-1} & m_{i+1 j}
\end{array}
$$

We start with proving that $c_{i j} \leq 0$ implies $c_{i+1 j} \leq 0$. If $c_{i j} \leq 0$ then $m_{i j}<(i+1)(j+1)$ (since otherwise $m_{r s}=(r+1)(s+1)$ for every $(r, s) \leq(i, j)$, and so $\left.c_{i j}=1\right)$, consequently $h_{i j}>0$. Our aim is to prove that $m_{i+1 j} \leq m_{i j}+m_{i+1 j-1}-m_{i j-1}$ or equivalently that

$$
h_{i+1 j}-h_{i+1 j-1}>h_{i j}-h_{i j-1}
$$

the conclusion will follow by induction. 
Let $L$ be a $(1,0)$-line and $L^{\prime}$ be a $(0,1)$-line such that $X \cap L=$ $X \cap L^{\prime}=\varnothing$, and the point $P=L \cap L^{\prime}$ is not in the base locus of $H^{0}\left(\mathscr{J}_{X}(i+1, j)\right)$. Consider the commutative diagram

$$
\begin{array}{cccc}
0 \rightarrow H^{0}\left(\mathscr{I}_{X}(i, j-1)\right) & \stackrel{\alpha}{\rightarrow} & H^{0}\left(\mathscr{I}_{X}(i, j)\right) & \rightarrow \text { Coker } \alpha \rightarrow 0 \\
\downarrow^{\beta} & \downarrow^{\prime} & \beta^{\prime \prime} \\
0 \rightarrow H^{0}\left(\mathscr{I}_{X}(i+1, j-1)\right) & \stackrel{\alpha}{\rightarrow} H^{0}\left(\mathscr{I}_{X}(i+1, j)\right) & \rightarrow \text { Coker } \alpha^{\prime} \rightarrow 0
\end{array}
$$

in which $\alpha$ and $\alpha^{\prime}$ are given by multiplication for $L^{\prime}, \beta$ and $\beta^{\prime}$ are given by multiplication for $L$, and $\beta^{\prime \prime}$ is the induced map. Since $\operatorname{dim} \operatorname{Coker} \alpha=h_{i j}-h_{i j-1}$ and $\operatorname{dim} \operatorname{Coker} \alpha^{\prime}=h_{i+1 j}-h_{i+1 j-1}$ it is enough to prove that $\beta^{\prime \prime}$ is injective but not surjective. Let $\bar{f} \in$ Coker $\alpha$ be a non-zero element: such an element exists since $H^{0}\left(\mathscr{I}_{X}(i, j)\right) \neq 0$ and $\alpha$ is not surjective; then $\bar{f}$ is the image of an element $f \in H^{0}\left(\mathscr{I}_{X}(i, j)\right)$ which does not contain $L^{\prime}$ as a factor. Now $\beta^{\prime \prime}(\bar{f}) \neq 0$ since $\beta^{\prime}(f)=f L \notin \operatorname{Im} \alpha^{\prime}$ by the choice of $f$.

To prove that $\beta^{\prime \prime}$ is not surjective observe that not any element in $H^{0}\left(\mathscr{I}_{X}(i+1, j)\right)$ is of the form $L f+L^{\prime} g$ with $f \in H^{0}\left(\mathscr{J}_{X}(i, j)\right)$ and $g \in H^{0}\left(\mathscr{J}_{X}(i+1, j-1)\right)$ : in fact $L f+L^{\prime} g$ vanishes at $P$ for every $f$ and $g$, while $P$ is not in the base locus of $H^{0}\left(\mathscr{J}_{X}(i+1, j)\right)$.

For (ii) it is sufficient to note that if for some $(i, j)$ we had $c_{i j}>1$, then by the first part of the proposition one would have $c_{r s} \geq 1$ for every $(r, s) \leq(i, j)$. Hence we would have $m_{i j}=\sum_{h \leq i, k \leq j} c_{h k}>$ $(i+1)(j+1)$, a contradiction.

REMARK 2.8. Let $M_{X}=\left(m_{i j}\right)$ be the Hilbert matrix of a 0-dimensional subscheme $X \subset Q$. By previous propositions the following terminology makes sense.

For every $i \geq 0$ we set

$$
j(i)=\min \left\{t \in \mathbf{N} \mid m_{i t}=m_{i t+1}\right\}=\min \left\{t \in \mathbf{N} \mid a_{i t+1}=0\right\},
$$

and for every $j \geq 0$ we set

$$
i(j)=\min \left\{t \in \mathbf{N} \mid m_{t j}=m_{t+1 j}\right\}=\min \left\{t \in \mathbf{N} \mid b_{t+1 j}=0\right\} .
$$

The sequences $i(j)$ and $j(i)$ are easily seen to be non-increasing (use the above propositions), and hence the meaningful part of the matrix $M_{X}$ sits inside the rectangle with opposite vertices $(0,0),(i(0), j(0))$; this means that for every $i>i(0)$ the $i$ th row is equal to the $i(0)$ th row, and for every $j>j(0)$ the $j$ th column is equal to the $j(0)$ th column. Of course for $(i, j) \geq(i(0), j(0)) m_{i j}=\operatorname{deg} X$, and outside the above rectangle $\Delta M_{X}$ has null entries. 
With this notation and with Theorem 1.2 in mind, one sees that $X$ is contained in a curve of type $(i(0)+1,0)$ and in a curve of type $(0, j(0)+1)$; therefore the minimal complete intersection containing $X$ is given by these two curves (see Remark 1.3).

REMARK 2.9. (i) One can represent the result of Proposition 2.7 just saying that each column of $\Delta^{R} M_{X}$ is a sequence of type $1,2, \ldots$, $t-1, t, t_{1}, t_{2}, \ldots$ in which $t \geq t_{1} \geq \cdots$, and $t_{i}=t_{i+1}$ for $i \gg 0$. The same holds for the rows of $\Delta^{C} M_{X}$.

(ii) In $\Delta M_{X}$ we have:

$c_{0 j}=\left\{\begin{array}{ll}1 & \text { for } 0 \leq j \leq j(0), \\ 0 & \text { otherwise }\end{array} \quad\right.$ and $c_{i 0}= \begin{cases}1 & \text { for } 0 \leq i \leq i(0), \\ 0 & \text { otherwise }\end{cases}$

(iii) Proposition 2.5 in terms of the matrix $\Delta M_{X}$ can be expressed as:

for every $(i, j) \quad 0 \leq \sum_{t=0}^{j} c_{i t} \leq \sum_{t=0}^{j} c_{i-1 t}:$ this means $b_{i j} \leq b_{i-1 j}$;

for every $(i, j) \quad 0 \leq \sum_{t=0}^{i} c_{t j} \leq \sum_{t=0}^{i} c_{t j-1}$ : this means $a_{i j} \leq a_{i j-1}$.

(iv) Propositions 2.5 and 2.7 give on the matrix $\Delta^{2} M_{X}=\left(d_{i j}\right)$ the following conditions:

(1) for every $i, \sum_{t \geq 0} d_{i t}=0$ and, for every $j, \sum_{t \geq 0} d_{t j}=0$; this because $c_{i j}=0$ for $i \gg 0$ or for $j \gg 0$;

(2)

$$
d_{i j}= \begin{cases}1 & \text { for } i=j=0, \\ 0 & \text { for } i=0 \text { and } j \neq j(0)+1 \text { or } j=0 \text { and } i \neq i(0)+1, \\ -1 & \text { for } i=0 \text { and } j=j(0)+1 \text { or } j=0 \text { and } i=i(0)+1\end{cases}
$$

(3) If $\sum_{r \leq i, s \leq j} d_{r s} \leq 0$ then $\sum_{r \leq i^{\prime}, s \leq j^{\prime}} d_{r s} \leq 0$ for $\left(i^{\prime}, j^{\prime}\right) \geq(i, j)$;

(4) for every $(i, j)$ we have by a straight computation:

$$
\begin{aligned}
& \sum_{t=0}^{j} c_{i t}=\sum_{s \leq j}\left[(s+1) \sum_{t \leq i} d_{t j-s}\right] \text { and } \\
& \sum_{t=0}^{i} c_{t j}=\sum_{s \leq i}\left[(s+1) \sum_{t \leq j} d_{i-s t}\right] ;
\end{aligned}
$$

so the inequalities in (iii) become:

$$
\sum_{s \leq j}\left[(s+1) \sum_{t \leq i} d_{t j-s}\right] \geq 0, \quad \text { and } \quad \sum_{s \leq i}\left[(s+1) \sum_{t \leq j} d_{i-s t}\right] \geq 0,
$$




$$
\sum_{s \leq j}(s+1) d_{i j-s} \leq 0, \quad \text { and } \quad \sum_{s \leq i}(s+1) d_{i-s j} \leq 0 .
$$

REMARK 2.10. When $Q$ is embedded in $\mathbf{P}^{3}$ then the sequence $m_{i i}$ is the Hilbert function of $X$ as a subscheme of $\mathbf{P}^{3}$ (see Remark 2.1). In this case, if $m_{i i}<(i+1)^{2}$ then $\Delta H F(X, i) \geq \Delta H F(X, i+1)$. In fact, by Proposition 2.5 we have $a_{i-1 i} \geq a_{i-1 i+1}$ and $b_{i i+1} \geq b_{i+1 i+1}$; by Proposition 2.7 and the hypothesis we have $b_{i i} \geq b_{i i+1}$. From these inequalities with a simple computation we get:

$$
\begin{aligned}
& m_{i-1 i+1}-m_{i-1 i} \leq m_{i-1 i}-m_{i-1 i-1} \quad \text { and } \\
& m_{i+1 i+1} \leq m_{i-1 i+1}+2 b_{i i}=m_{i-1 i+1}+2 m_{i i}-2 m_{i-1 i}
\end{aligned}
$$

summing up we obtain $m_{i+1 i+1}+m_{i-1 i-1} \leq 2 m_{i i}$, i.e. $m_{i+1 i+1}-m_{i i} \leq$ $m_{i i}-m_{i-1 i-1}$.

This result was recently proved, by different methods, in [R1].

TheOREM 2.11. Let $X \subset Q$ be a 0-dimensional subscheme, then its Hilbert matrix $M_{X}=\left(m_{i j}\right)$ is admissible.

Proof. Just apply Propositions 2.5 and 2.7.

Now we will give some geometric information contained in the Hilbert matrix of a 0 -dimensional subscheme of $Q$.

As a prelude to the next theorem, let us look at the following example. Let $X \subset Q$ be a set of 16 points with Hilbert matrix $M_{X}$, of size $(3,4)$ :

\begin{tabular}{r|r|r|r|r|r|r|} 
& \multicolumn{2}{|c}{0} & \multicolumn{1}{c}{1} & \multicolumn{1}{c}{2} & \multicolumn{1}{c}{3} & \multicolumn{1}{c}{4} \\
\hline 0 & 1 & 2 & 3 & 4 & 5 & 5 \\
\cline { 2 - 7 } 1 & 2 & 4 & 6 & 8 & 10 & 10 \\
\cline { 2 - 7 } 2 & 3 & 6 & 9 & 12 & 14 & 14 \\
\cline { 2 - 7 } 3 & 4 & 8 & 11 & 14 & 16 & 16 \\
\cline { 2 - 7 } 4 & 4 & 8 & 11 & 14 & 16 & 16 \\
\cline { 2 - 7 }
\end{tabular}

$a$

If one writes down the matrices $\Delta^{R} M_{X}$ and $\Delta^{C} M_{X}$ and uses the next theorem, one sees that there are two lines of type $(1,0)$ each 
containing 5 points, one with 4 points and one with 2 points; similarly there are two lines of type $(0,1)$ each containing four points, two more lines with 3 points, and one with 2 points.

Moreover, in this particular example, the same thing can be seen more easily looking directly at the matrix $\Delta M_{X}$

\begin{tabular}{|c|c|c|c|c|c|c|}
\hline & 0 & 1 & 2 & 3 & 4 & 5 \\
\hline 0 & 1 & 1 & 1 & 1 & 1 & 0 \\
\hline 1 & 1 & 1 & 1 & 1 & 1 & 0 \\
\hline 2 & 1 & 1 & 1 & 1 & 0 & 0 \\
\hline 3 & 1 & 1 & 0 & 0 & 0 & 0 \\
\hline 4 & 0 & 0 & 0 & 0 & 0 & 0 \\
\hline
\end{tabular}

$a$

and counting the number of " 1 's" in each row and column (see $\S 4$ ).

What we are saying for points on the quadric makes sense also for any 0-dimensional subscheme of $Q$. We need to explain what " $n$ points on a line" means for non-reduced subschemes.

Let $X$ be any 0-dimensional subscheme of $Q$ and $I=I(X) \subset S$ be its homogeneous saturated ideal. For any homogeneous form $f \in S$ consider the ideal $(I, f)$ : this is not in general a saturated ideal, anyway denote by $Y$ the subscheme of $X$ that it defines. Then the residual subscheme of $Y$ in $X$ is defined by the ideal $I: f$, which is saturated as one can see by a standard check.

Since $I(X)$ is saturated, it contains a form $f\left(u, u^{\prime}\right) \otimes 1$ of degree $(n, 0)$ for some $n$ (see Theorem 1.2). Let $f\left(u, u^{\prime}\right)=\prod_{i=1}^{r}\left(a_{i} u+b_{i} u^{\prime}\right)^{s_{i}}$ be the decomposition of $f\left(u, u^{\prime}\right)$, and set $a_{i} u+b_{i} u^{\prime}=u_{i} \quad(i=$ $1,2, \ldots, r)$. The line $u_{i}$ appears with multiplicity $s_{i}$ in the decomposition of $f$; we count the number of "points of $X$ " on each copy of $u_{i}$ in the following way:

$$
\text { set } \begin{aligned}
J_{1} & =\left(I, u_{i}\right) \text { and } I_{1}=I: u_{i} ; \\
J_{2} & =\left(I_{1}, u_{i}\right) \text { and } I_{2}=I_{1}: u_{i} ; \\
\ldots \ldots \ldots \ldots \ldots \ldots \ldots \ldots \ldots \ldots & \ldots \ldots \ldots \\
J_{s_{i}} & =\left(I_{s_{i}-1}, u_{i}\right) \text { and } I_{s_{i}}=I_{s_{i}-1}: u_{i}
\end{aligned}
$$

( $I_{s_{i}}$ is not supported at any point of $u_{i}$ ). 
Now the "first" copy of $u_{i}$ contains $\operatorname{deg}\left(\right.$ sat $\left.J_{1}\right)$ points of $X, \ldots$, the "last one" contains $\operatorname{deg}\left(\operatorname{sat} J_{S_{l}}\right)$ points of $X$.

In the next theorem we shall use the following property (Bézout): with the above notation let $g \in S$ be any irreducible form of degree $(a, b)$ and $h \in H^{0}\left(\mathscr{I}_{X}(c, d)\right)$. If $\operatorname{deg}(\operatorname{sat}(I, g))>a d+b c$ then $h=g g^{\prime}$ for some $g^{\prime}$.

Theorem 2.12. Let $X \subset Q$ be a 0-dimensional subscheme, and $M_{X}=\left(m_{i j}\right)$ its Hilbert matrix. Then for every $j \geq 0$ there are just $a_{i(0) j}-a_{i(0) j+1}$ lines of type $(1,0)$ each containing just $j+1$ points of $X$ and, similarly, for every $i \geq 0$ there are just $b_{i j(0)}-b_{i+1 j(0)}$ lines of type $(0,1)$ each containing just $i+1$ points of $X$.

Proof. We establish the theorem for the $(1,0)$-lines; one could work in a similar way for the other lines. We proceed by induction on $j$. Let us consider the following inductive hypothesis: there are just

(1)

$$
\left\{\begin{array}{l}
r_{1}=a_{i(0) 0}-a_{i(0) 1}(1,0) \text {-lines containing just } 1 \text { point of } X \\
r_{2}=a_{i(0) 1}-a_{i(0) 2}(1,0) \text {-lines containing just } 2 \text { points of } X \\
\ldots \ldots \ldots \ldots \ldots \ldots \ldots \ldots \ldots \ldots \ldots \ldots \ldots \ldots \ldots \ldots \ldots \ldots \ldots \ldots \ldots \ldots \ldots \ldots \ldots \ldots \ldots \ldots \ldots \ldots \ldots \ldots \ldots \ldots \ldots \\
r_{j}=a_{i(0) j-1}-a_{i(0) j}(1,0) \text {-lines containing just } j \text { points of } X
\end{array}\right.
$$

As the hypothesis (1) is empty for $j=0$, we need deal only with the general case. Denote by $r_{j+1}$ the number of $(1,0)$-lines containing just $j+1$ points of $X$.

Since $X$ is contained in $i(0)+1(1,0)$-lines, by hypothesis $(1)$ there are

$$
\delta=i(0)+1-\sum_{t=1}^{j+1} r_{t}
$$

lines containing more than $j+1$ points of $X$; therefore every element of $H^{0}\left(\mathscr{I}_{X}(i(0), j+1)\right)$ is the union of a fixed curve $f$ of degree $(\delta, 0)$ ( $\delta$ fixed lines when $X$ is reduced) and a curve of type $(i(0)-\delta, j+1)$ passing through $X^{\prime}$, where $X^{\prime} \subset X$ is the subscheme defined by $I(X): f$ (when $X$ is reduced $X^{\prime}$ is the subset of points in $X$ lying on the remaining lines); of course $\operatorname{deg} X^{\prime}=\sum_{t=1}^{j+1} t r_{t}$.

Claim. $X^{\prime}$ imposes independent conditions on $H^{0}(i(0)-\delta, j+1)$.

We show that $m_{i(0)-\delta j}^{\prime}=\operatorname{deg} X^{\prime}$ where $M_{X^{\prime}}=\left(m_{i j}^{\prime}\right)$ denotes the Hilbert matrix of $X^{\prime}$. 
Observe first that for $t \leq j+1$, by definition of $X^{\prime}$ one has:

$$
\begin{aligned}
m_{i(0) t} & =(i(0)+1)(t+1)-h^{0}\left(\mathscr{I}_{X}(i(0), t)\right) \\
& =(i(0)+1-\delta)(t+1)-h^{0}\left(\mathscr{I}_{X^{\prime}}(i(0)-\delta, t)\right)+\delta(t+1) \\
& =m_{i(0)-\delta t}^{\prime}+\delta(t+1) .
\end{aligned}
$$

Since by (1), for every $p \leq j$, we have $r_{p}+\cdots+r_{j}=a_{i(0) p-1}-a_{i(0) j}$, we can compute:

$$
\begin{aligned}
\operatorname{deg} X^{\prime}= & \sum_{t=1}^{j+1} t r_{t}=\left(r_{1}+\cdots+r_{j}\right)+\left(r_{2}+\cdots+r_{j}\right) \\
& +\cdots+r_{j}+(j+1) r_{j+1} \\
= & \left(a_{i(0) 0}-a_{i(0) j}\right)+\left(a_{i(0) 1}-a_{i(0) j}\right) \\
& +\cdots+\left(a_{i(0) j-1}-a_{i(0) j}\right)+(j+1) r_{j+1} \\
= & m_{i(0) j}-(j+1)\left(a_{i(0) j}-r_{j+1}\right) .
\end{aligned}
$$

Again by (1) one gets:

$$
\begin{aligned}
a_{i(0) j} & =a_{i(0) j-1}-r_{j}=a_{i(0) j-2}-r_{j-1}-r_{j}=\cdots=a_{i(0) 0}-r_{1}-\cdots-r_{j} \\
& =i(0)+1-r_{1}-\cdots-r_{j} .
\end{aligned}
$$

By substituting in (2) we have

$$
\begin{aligned}
\operatorname{deg} X^{\prime} & =m_{i(0) j}-(j+1)\left(i(0)+1-\sum_{t=1}^{j+1} r_{t}\right) \\
& =m_{i(0) j}-\delta(j+1)=m_{i(0)-\delta j}^{\prime} .
\end{aligned}
$$

Now, since

$$
\begin{aligned}
H^{0}\left(\mathscr{I}_{X}(i(0), j+1)\right) & \cong H^{0}\left(\mathscr{J}_{X^{\prime}}(i(0)-\delta, j+1)\right) \text { and } \\
i(0)-\delta+1 & =\sum_{t=1}^{j+1} r_{t},
\end{aligned}
$$

by the claim we have:

$$
\begin{aligned}
m_{i(0) j+1} & =(i(0)+1)(j+2)\left[\left(\sum_{t=1}^{j+1} r_{t}\right)(j+2)-\sum_{t=1}^{j+1} t r_{t}\right] \\
& =(i(0)+1)(j+2)-\sum_{t=1}^{j+1}(j+2-t) r_{t}
\end{aligned}
$$


on the other hand, for every $s \leq j$, summing up the relations in (1), we have

$$
\sum_{t=1}^{s} r_{t}=a_{i(0) 0}-a_{i(0) s}=i(0)+1-a_{i(0) s} ;
$$

so by definition of $a_{i j}$ we get:

$$
\begin{aligned}
m_{i(0) j} & =i(0)+1+\sum_{s=1}^{j} a_{i(0) s}=i(0)+1+\sum_{s=1}^{j}\left[(i(0)+1)-\sum_{t=1}^{s} r_{t}\right] \\
& =(i(0)+1)(j+1)-\sum_{s=1}^{j}(j-s+1) r_{s} .
\end{aligned}
$$

Finally, we get

$$
\begin{aligned}
a_{i(0) j+1}= & m_{i(0) j+1}-m_{i(0) j} \\
= & (i(0)+1)(j+2)-\sum_{t=1}^{j+1}(j+2-t) r_{t} \\
& -(i(0)+1)(j+1)+\sum_{t=1}^{j}(j+1-t) r_{t} \\
= & i(0)+1-\sum_{t=1}^{j} r_{t}-r_{j+1}=a_{i(0) j}-r_{j+1} .
\end{aligned}
$$

COROLLARY 2.13. With the hypotheses of the above theorem, every linear system of curves of type $(i, j)$ passing through $X$, with $i \leq i^{*}=$ $\min \left\{t \in N \mid m_{t j(t)}=\operatorname{deg} X\right\}\left(\right.$ resp. $\left.j \leq j^{*}=\min \left\{t \in N \mid m_{i(t) t}=\operatorname{deg} X\right\}\right)$ has at least one fixed line of type $(0,1)$ (resp. of type $(1,0))$.

Proof. By minimality on $i^{*}$, in the matrix $\Delta^{C} M_{X}$ we have $b_{i^{*}+1 j\left(i^{*}\right)}$ $=0$ and $b_{i^{*} j\left(i^{*}\right)}>0$. Note that $b_{i^{*} j(0)}>0$ because $m_{i^{*} j(0)}=\operatorname{deg} X$ and $m_{i^{*}-1 j(0)}<\operatorname{deg} X$.

Applying the previous theorem one sees that there are $b_{i^{*} j(0)}(0,1)$ lines containing $i^{*}+1$ points of $X$. Every curve of type $(i, j)$ passing through $X$, with $i \leq i^{*}$, will contain such lines. One can repeat the same argument starting with $\Delta^{R} M_{X}$.

EXAMPLE 2.14. Not every admissible matrix is the Hilbert matrix of some 0 -dimensional subscheme of $Q$. The following admissible matrix explains this situation: 


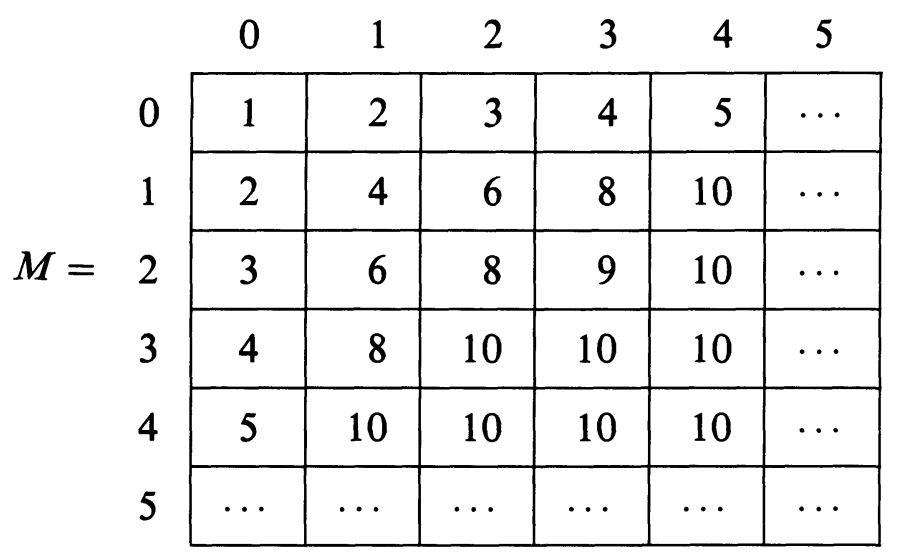

We want to show that there is no set of 10 points $X \subset Q$ such that $M=M_{X}$. By Theorem 2.12 such an $X$ would belong to $5(1,0)$ lines $L_{i}$ and to $5(0,1)$-lines $L_{i}^{\prime}, 2$ points of $X$ on each of these lines. Looking at $M$ one sees that $h^{0}\left(\mathscr{I}_{X}(2,3)\right)=3$; therefore there would exist a curve $C$ of type $(2,3)$ passing through $X$ and containing one of the above lines as a component, say $L_{1}$ (take 2 further points on $L_{1}$ and remark that the dimension of the linear system of curves of type $(2,3)$ through $X$ and these two points is $\geq 1)$. Hence, $C=L_{1} \cdot C^{\prime}$ where $C^{\prime}$ is a curve of type $(1,3)$ containing the 8 points $X-\left\{L_{1} \cap X\right\}$. Now the intersection on $Q$ gives $(1,3) \cdot(0,1)=1$, so $C^{\prime}$ must contain as components three lines $L_{i}^{\prime}$ (each with 2 points of $X)$ and another line of type $(1,0)$ passing through the remaining two points: so, these two points together with the two points on $L_{1}$ form a complete intersection $(0,2),(2,0)$; but this is impossible because we can repeat the argument on each line $L_{i}$ (the number of the $L_{i}$ is odd).

LEMMA 2.15. Let $X \subset Q$ be a 0 -dimensional subscheme, and $M_{X}=$ $\left(m_{i j}\right)$ its Hilbert matrix; let $\Delta M_{X}$ be of size $(a, b)$ and $L_{0}^{\prime}, L_{1}^{\prime}, \ldots$, $L_{b}^{\prime}$ be the $(0,1)$-lines containing $X$. Take any $(1,0)$-line $L$ disjoint from $X$ and consider $Z=X \cup Y$, where $Y=L \cap\left(\bigcup_{i=0}^{n} L_{i}^{\prime}\right)$ with $n \geq b$ and $L_{b+1}^{\prime}, \ldots, L_{n}^{\prime}$ arbitrary $(0,1)$-lines.

Then the Hilbert matrix of $Z, M_{Z}=\left(m_{i j}^{\prime}\right)$ is the following.

$$
\begin{aligned}
& m_{0 j}^{\prime}= \begin{cases}j+1 & \text { for } 0 \leq j \leq n, \\
n+1 & \text { for } j>n\end{cases} \\
& m_{i+1 j}^{\prime}= \begin{cases}m_{i j}+j+1 & \text { for } i \geq 0,0 \leq j \leq n, \\
m_{i j}+n+1 & \text { for } i \geq 0, j>n .\end{cases}
\end{aligned}
$$


Proof. One can express the lemma in terms of the first difference matrices, $\Delta M_{X}=\left(c_{i j}\right), \Delta M_{Z}=\left(c_{i j}^{\prime}\right)$ :

$$
\begin{gathered}
c_{0 j}^{\prime}= \begin{cases}1 & \text { for } 0 \leq j \leq n, \\
0 & \text { for } j>n ;\end{cases} \\
c_{i+1 j}^{\prime}=c_{i j} \text { for }(i, j) \geq(0,0),
\end{gathered}
$$

which mean that $\Delta M_{Z}$ is obtained from $\Delta M_{X}$ just adding a 1 st row consisting of $n+1$ " 1 " entries.

We prove (2), as (1) is trivial. Observe that, for $j \leq n$, one has

$$
h^{0}\left(\mathscr{I}_{X}(i, j)\right)=h^{0}\left(\mathscr{I}_{Z}(i+1, j)\right)
$$

since every curve of type $(i+1, j)$ through $Z$ splits into $L$ and a curve of type $(i, j)$ through $X$; hence

$$
m_{i+1 j}^{\prime}=(i+2)(j+1)-h^{0}\left(\mathscr{J}_{Z}(i+1, j)\right)=m_{i j}+j+1 .
$$

When $j>n$ we have $c_{i+1 j}^{\prime}=c_{i j}=0$ and we are done.

Of course a similar result can be proved adding $n+1$ points on a $(0,1)$-line $L^{\prime}$ disjoint from $X$.

COROLlary 2.16. With the same hypotheses of the above theorem, if the $(0,1)$-line $L_{0}^{\prime}$ contains $a+1$ points of $X$, then $X^{\prime}=X-\left\{L_{0}^{\prime} \cap X\right\}$ has the following Hilbert matrix:

$$
\Delta M_{X^{\prime}}(i, j)=\Delta M_{X}(i, j+1) \quad(i, j) \geq(0,0) .
$$

Proof. Note that $X=X^{\prime} \cup Y$, where $Y=L_{0}^{\prime} \cap X$, and apply Lemma 2.15 changing rows with columns.

3. The resolution of the ideal sheaf $\mathscr{I}_{X}$. Let $X \subset Q$ be a 0 dimensional subscheme and $I(X) \subset S$ the saturated ideal of $X$. Note that $1 \leq \operatorname{depth} S(X) \leq 2$ : in fact $I(X)$ contains an $S$-sequence of length 2, and in $S(X)$ there is a regular element (it is enough to take an element of $S$ which does not vanish at any point of $X$ ). Therefore $I(X)$ has an $S$-free minimal resolution of length $\leq 3$ with morphisms of degree $(0,0)$. If this resolution has length 2 , i.e. when $\operatorname{depth} S(X)=2$, then $S(X)$ is a Cohen-Macaulay ring and $X$ is called arithmetically Cohen-Macaulay (ACM for short).

EXAMPLE 3.1. Although $X$ has maximal codimension in $Q$, it is not always true that $S(X)$ is Cohen-Macaulay, in opposition to what happens for subschemes of maximal codimension in $\mathbf{P}^{n}$.

Here is a simple example of this fact. 
Take on $Q$ two non-collinear points (i.e. not contained on a line of $Q)$, say $P_{1}, P_{2}$, and let $\mathfrak{p}_{1}=(u \otimes 1,1 \otimes v)$ and $\mathfrak{p}_{2}=\left(u^{\prime} \otimes 1,1 \otimes v^{\prime}\right)$ their defining ideals. If $X=\left\{P_{1}, P_{2}\right\}$ one gets $I(X)=\left(u u^{\prime} \otimes 1\right.$, $\left.u \otimes v^{\prime}, u^{\prime} \otimes v, 1 \otimes v v^{\prime}\right)$. One sees that $\left(u+u^{\prime}\right) \otimes 1$ is regular in $S(X)$; let us check that depth $S / J=0$, where $J=\left(I(X),\left(u+u^{\prime}\right) \otimes 1\right)$. In fact, in $S / J$ the homogeneous elements are either of type $u \otimes$ $g\left(v, v^{\prime}\right)$ or $1 \otimes h\left(v, v^{\prime}\right)$, where $g\left(v, v^{\prime}\right)$ and $h\left(v, v^{\prime}\right)$ are forms and $\operatorname{deg} h\left(v, v^{\prime}\right)>0$. They are both annihilated by $u \otimes 1$. So, $\operatorname{depth} S(X)=1<\operatorname{dim} S(X)$.

Of course, two collinear points are complete intersection, hence ACM. In $\S 4$ we will see that not every ACM 0-dimensional subscheme of $Q$ is c.i.

Let

$$
\begin{aligned}
0 & \rightarrow \bigoplus_{i=1}^{p} S\left(-a_{3 i},-a_{3 i}^{\prime}\right) \rightarrow \bigoplus_{i=1}^{n} S\left(-a_{2 i},-a_{2 i}^{\prime}\right) \\
& \rightarrow \bigoplus_{i=1}^{m} S\left(-a_{1 i},-a_{1 i}^{\prime}\right) \rightarrow I(X) \rightarrow 0
\end{aligned}
$$

be the minimal free resolution of the saturated ideal $I(X)$, with morphisms of degree $(0,0)$. From this, taking sheaves, one gets an $\mathscr{O}_{Q^{-}}$ free resolution of the ideal sheaf $\mathscr{J}_{X}$.

Take now any $\mathscr{O}_{Q}$-free minimal resolution of $\mathscr{I}_{X}$

$$
0 \rightarrow \mathscr{L}_{2} \rightarrow \mathscr{L}_{1} \stackrel{\varphi}{\longrightarrow} \mathscr{L}_{0} \rightarrow \mathscr{I}_{X} \rightarrow 0
$$

such that

$(*)\left\{\begin{array}{rr}\text { for any }(r, s) & H^{0}\left(\mathscr{L}_{0}(r, s)\right) \rightarrow H^{0}\left(\mathscr{I}_{X}(r, s)\right) \text { is surjective, } \\ \text { for any }(r, s) & H^{0}\left(\mathscr{L}_{1}(r, s)\right) \rightarrow H^{0}(\mathscr{E}(r, s)) \text { is surjective, } \\ \text { with } \mathscr{E}=\operatorname{Im} \varphi\end{array}\right.$

With this choice, for every $(r, s)$ we obtain the exact sequence

$$
\begin{aligned}
0 & \rightarrow H^{0}\left(\mathscr{L}_{2}(r, s)\right) \rightarrow H^{0}\left(\mathscr{L}_{1}(r, s)\right) \rightarrow H^{0}\left(\mathscr{L}_{0}(r, s)\right) \\
& \rightarrow H^{0}\left(\mathscr{I}_{X}(r, s)\right) \rightarrow 0
\end{aligned}
$$

and since $H_{*}^{0}\left(\mathscr{J}_{X}\right)=\bigoplus_{r \geq 0, s \geq 0} H^{0}\left(\mathscr{F}_{X}(r, s)\right) \cong I(X)$, taking sums on $(r, s)$ we obtain a resolution which is isomorphic to (1). Thus the 
resolution

$$
\begin{aligned}
0 & \rightarrow \bigoplus_{i=1}^{p} \mathscr{O}_{Q}\left(-a_{3 i},-a_{3 i}^{\prime}\right) \rightarrow \bigoplus_{i=1}^{n} \mathscr{O}_{Q}\left(-a_{2 i},-a_{2 i}^{\prime}\right) \\
& \rightarrow \bigoplus_{i=1}^{m} \mathscr{O}_{Q}\left(-a_{1 i},-a_{1 i}^{\prime}\right) \rightarrow \mathscr{I}_{X} \rightarrow 0
\end{aligned}
$$

obtained by taking sheaves in (1), satisfies conditions $(*)$.

From now on, we will refer to (2) as the minimal free resolution of $\mathscr{I}_{X}$ without further specification.

The convenience of this choice is clear since from (2) one can compute $h^{0}\left(\mathscr{I}_{X}(r, s)\right)$ for every $(r, s) \geq(0,0)$ :

$$
\begin{aligned}
h^{0}\left(\mathscr{I}_{X}(r, s)\right)= & \sum_{i=1}^{m} h^{0}\left(r-a_{1 i}, s-a_{1 i}^{\prime}\right)-\sum_{i=1}^{n} h^{0}\left(r-a_{2 i}, s-a_{2 i}^{\prime}\right) \\
& +\sum_{i=1}^{p} h^{0}\left(r-a_{3 i}, s-a_{3 i}^{\prime}\right) \\
= & \sum_{i=1}^{m}\left(r-a_{1 i}+1\right)_{+}\left(s-a_{1 i}^{\prime}+1\right)_{+} \\
& -\sum_{i=1}^{n}\left(r-a_{2 i}+1\right)_{+}\left(s-a_{2 i}^{\prime}+1\right)_{+} \\
& +\sum_{i=1}^{p}\left(r-a_{3 i}+1\right)_{+}\left(s-a_{3 i}^{\prime}+1\right)_{+}
\end{aligned}
$$

where for every $h \in \mathbf{Z}$ we mean $h_{+}=\max \{h, 0\}$.

REMARK 3.2. We took great care in defining the resolution of $\mathscr{I}_{X}$, since, contrary to the situation of sheaves on $\mathbf{P}^{n}$, on $Q$ it may happen that the ideal sheaf $\mathscr{I}_{X}$ of a 0 -dimensional subscheme $X \subset Q$ has a minimal free resolution of length 2

$$
0 \rightarrow \mathscr{L}_{1} \rightarrow \mathscr{L}_{0} \rightarrow \mathscr{J}_{X} \rightarrow 0
$$

without $X$ being ACM. This happens because the map $H_{*}^{0}\left(\mathscr{L}_{0}\right) \rightarrow$ $H_{*}^{0}\left(\mathscr{J}_{X}\right)$ could be nonsurjective. This is the case, for instance, when $X$ is ideally a complete intersection, i.e. when there exists a sheaf surjection $\mathscr{O}_{Q}^{\oplus 2} \rightarrow \mathscr{J}_{X}$, but $X$ is not c.i. (see Example 3.1).

With the notation of resolution (2), we set the following:

$$
\begin{aligned}
\alpha_{h k} & =\#\left\{\left(a_{1 i}, a_{1 i}^{\prime}\right)=(h, k)\right\}, \\
\beta_{h k} & =\#\left\{\left(a_{2 i}, a_{2 i}^{\prime}\right)=(h, k)\right\}, \\
\gamma_{h k} & =\#\left\{\left(a_{3 i}, a_{3 i}^{\prime}\right)=(h, k)\right\} .
\end{aligned}
$$


Proposition 3.3. Let $X \subset Q$ be a 0-dimensional subscheme and let

$$
\begin{aligned}
0 & \rightarrow \bigoplus_{i=1}^{p} \mathscr{O}_{Q}\left(-a_{3 i},-a_{3 i}^{\prime}\right) \rightarrow \bigoplus_{i=1}^{n} \mathscr{Q}_{Q}\left(-a_{2 i},-a_{2 i}^{\prime}\right) \\
\stackrel{\varphi}{\rightarrow} \bigoplus_{i=1}^{m} \mathscr{Q}_{Q}\left(-a_{1 i},-a_{1 i}^{\prime}\right) & \rightarrow \mathscr{I}_{X} \rightarrow 0
\end{aligned}
$$

be the minimal free resolution of $\mathscr{I}_{X}$. Then we have:

(i) $n+1=m+p$

(ii) $\sum_{i=1}^{m} a_{1 i}-\sum_{i=1}^{n} a_{2 i}+\sum_{i=1}^{p} a_{3 i}=\sum_{i=1}^{m} a_{1 i}^{\prime}-\sum_{i=1}^{n} a_{2 i}^{\prime}+\sum_{i=1}^{p} a_{3 i}^{\prime}=$ 0 ;

(iii) $\operatorname{deg} X=-\sum_{i=1}^{m} a_{1 i} a_{1 i}^{\prime}+\sum_{i=1}^{n} a_{2 i} a_{2 i}^{\prime}-\sum_{i=1}^{p} a_{3 i} a_{3 i}^{\prime}$;

(iv) for every $i=1,2, \ldots, m$ there exists $j(1 \leq j \leq n)$ such that $\left(a_{2 j}, a_{2 j}^{\prime}\right)>\left(a_{1 i}, a_{1 i}^{\prime}\right)$

(v) if a first syzygy exists, say of degree $\left(a_{2 r}, a_{2 r}^{\prime}\right)$, which is maximal with respect to the property " $\left(a_{2 r}, a_{2 r}^{\prime}\right) \nless\left(a_{3 i}, a_{3 i}^{\prime}\right)$ for all $i=$ $1,2, \ldots, p "$, then $h^{1}\left(\mathscr{I}_{X}\left(a_{2 r}-2, a_{2 r}^{\prime}-2\right)\right) \neq 0$. In this case, if $M_{X}$ is the Hilbert matrix of $X$, we have $M_{X}\left(a_{2 r}-2, a_{2 r}^{\prime}-2\right)<\operatorname{deg} X$;

(vi) the following relations between the given resolution of $\mathscr{I}_{X}$ and the matrices $M_{X}=\left(m_{i j}\right), \Delta M_{X}=\left(c_{i j}\right), \Delta^{2} M_{X}=\left(d_{i j}\right)$ hold:

$$
\begin{aligned}
m_{r s} & =(r+1)(s+1)-\sum_{\substack{h \leq r \\
k \leq s}}(r+1-h)(s+1-k)\left(\alpha_{h k}-\beta_{h k}+\gamma_{h k}\right), \\
c_{r s} & =1-\sum_{\substack{h \leq r \\
k \leq s}}\left(\alpha_{h k}-\beta_{h k}+\gamma_{h k}\right),
\end{aligned}
$$

$d_{00}=1, \quad$ and for every $(r, s)>(0,0) d_{r s}=-\alpha_{r s}+\beta_{r s}-\gamma_{r s} ;$

(vii) if $\Delta M_{X}$ is of size $(a, b)$ then for every $(i, j) \geq(a+2, b+2)$ one has $\alpha_{i j}=\beta_{i j}=\gamma_{i j}=0$.

Proof. (i) and (ii) are well-known consequences of the exactness of the resolution. For (iii) we need an explicit computation. Since for $(r, s) \gg(0,0), m_{r s}=\operatorname{deg} X$, taking in mind the computation of 
$h^{0}\left(\mathscr{J}_{X}(r, s)\right)$ we have:

$$
\begin{aligned}
\operatorname{deg} X= & m_{r s}=(r+1)(s+1)-\sum_{i=1}^{m}\left(r-a_{1 i}+1\right)\left(s-a_{1 i}^{\prime}+1\right) \\
& +\sum_{i=1}^{n}\left(r-a_{2 i}+1\right)\left(s-a_{2 i}^{\prime}+1\right)-\sum_{i=1}^{p}\left(r-a_{3 i}+1\right)\left(s-a_{3 i}^{\prime}+1\right) \\
= & \sum_{i=1}^{m}\left[(s+1) a_{1 i}+(r+1) a_{1 i}^{\prime}-a_{1 i} a_{1 i}^{\prime}\right] \\
& -\sum_{i=1}^{n}\left[(s+1) a_{2 i}+(r+1) a_{2 i}^{\prime}-a_{2 i} a_{2 i}^{\prime}\right] \\
& +\sum_{i=1}^{p}\left[(s+1) a_{3 i}+(r+1) a_{3 i}^{\prime}-a_{3 i} a_{3 i}^{\prime}\right] \\
= & (s+1)\left[\sum_{i=1}^{m} a_{1 i}-\sum_{i=1}^{n} a_{2 i}+\sum_{i=1}^{p} a_{3 i}\right] \\
& +(r+1)\left[\sum_{i=1}^{m} a_{1 i}^{\prime}-\sum_{i=1}^{n} a_{2 i}^{\prime}+\sum_{i=1}^{p} a_{3 i}^{\prime}\right] \\
& -\sum_{i=1}^{m} a_{1 i} a_{1 i}^{\prime}+\sum_{i=1}^{n} a_{2 i} a_{2 i}^{\prime}-\sum_{i=1}^{p} a_{3 i} a_{3 i}^{\prime}
\end{aligned}
$$

now the conclusion follows using (ii). Notice that in the first equality we used (i).

To prove (iv) observe that if one generator of degree $\left(a_{1 r}, a_{1 r}^{\prime}\right)$ contradicts (iv), then the matrix of $\varphi$ would have the $r$ th row with all zeros: this would mean that the mentioned generator has no syzygies at all (not even the trivial one!).

(v) Splitting the resolution of $\mathscr{I}_{X}$ we have the exact sequences

$$
0 \rightarrow \mathscr{E} \rightarrow \bigoplus_{i=1}^{m} \mathscr{O}_{Q}\left(-a_{1 i},-a_{1 i}^{\prime}\right) \rightarrow \mathscr{I}_{X} \rightarrow 0,
$$

$$
0 \rightarrow \bigoplus_{i=1}^{p} \mathscr{O}_{Q}\left(-a_{3 i},-a_{3 i}^{\prime}\right) \rightarrow \bigoplus_{i=1}^{n} \mathscr{O}_{Q}\left(-a_{2 i},-a_{2 i}^{\prime}\right) \rightarrow \mathscr{E} \rightarrow 0
$$

where $\mathscr{E}=\operatorname{Im} \varphi$ is a locally free sheaf. Twisting in (4) by $\left(a_{2 r}-2\right.$, $\left.a_{2 r}^{\prime}-2\right)$, taking cohomology, using the minimality of the resolution and the hypothesis on $\left(a_{2 r}, a_{2 r}^{\prime}\right)$, one has $H^{2}\left(\mathscr{E}\left(a_{2 r}-2, a_{2 r}^{\prime}-2\right)\right) \neq 0$. 
Twisting (3) by the same degree and taking cohomology, we have

$$
\begin{aligned}
\cdots & \rightarrow H^{1}\left(\mathscr{I}_{X}\left(a_{2 r}-2, a_{2 r}^{\prime}-2\right)\right) \rightarrow H^{2}\left(\mathscr{E}\left(a_{2 r}-2, a_{2 r}^{\prime}-2\right)\right) \\
& \rightarrow H^{2}\left(\bigoplus_{i=1}^{m} \mathscr{O}_{Q}\left(a_{2 r}-2-a_{1 i}, a_{2 r}^{\prime}-2-a_{1 i}^{\prime}\right)\right) \rightarrow \cdots .
\end{aligned}
$$

Since the last term of this sequence vanishes because of the maximality assumption on $\left(a_{2 r}, a_{2 r}^{\prime}\right)$ and by (iv), we obtain

$$
H^{1}\left(\mathscr{I}_{X}\left(a_{2 r}-2, a_{2 r}^{\prime}-2\right)\right) \neq 0 \text {. }
$$

The second part is proven recalling that, for every $(i, j)$, $h^{1}\left(\mathscr{J}_{X}(i, j)\right)=\operatorname{deg} X-m_{i j}$.

(vi) Since for every $(r, s)$,

$$
\begin{aligned}
m_{r s}= & (r+1)(s+1)-\sum_{i=1}^{m}\left(r-a_{1 i}+1\right)_{+}\left(s-a_{1 i}^{\prime}+1\right)_{+} \\
& +\sum_{i=1}^{n}\left(r-a_{2 i}+1\right)_{+}\left(s-a_{2 i}^{\prime}+1\right)_{+} \\
& -\sum_{i=1}^{p}\left(r-a_{3 i}+1\right)_{+}\left(s-a_{3 i}^{\prime}+1\right)_{+}
\end{aligned}
$$

the first claim follows by definition of $\alpha_{h k}, \beta_{h k}, \gamma_{h k}$ and a straightforward computation. To compute $c_{r s}$ we employ the matrix $\Delta^{R} M_{X}=$ $\left(a_{r s}\right)$.

$$
\begin{aligned}
a_{r s}= & m_{r s}-m_{r s-1}=r+1-\sum_{\substack{h \leq r \\
k \leq s-1}}(r+1-h)\left(\alpha_{h k}-\beta_{h k}+\gamma_{h k}\right) \\
& -\sum_{h \leq r}(r+1-h)\left(\alpha_{h s}-\beta_{h s}+\gamma_{h s}\right) \\
= & r+1-\sum_{\substack{h \leq r \\
k \leq s}}(r+1-h)\left(\alpha_{h k}-\beta_{h k}+\gamma_{h k}\right) .
\end{aligned}
$$

Using the analogue expression for $a_{r-1 s}$, one gets

$$
\begin{aligned}
c_{r s} & =a_{r s}-a_{r-1 s} \\
& =1-\sum_{k \leq s}\left(\alpha_{r k}-\beta_{r k}+\gamma_{r k}\right)-\sum_{\substack{h \leq r-1 \\
k \leq s}}\left(\alpha_{h k}-\beta_{h k}+\gamma_{h k}\right) \\
& =1-\sum_{\substack{h \leq r \\
k \leq s}}\left(\alpha_{h k}-\beta_{h k}+\gamma_{h k}\right) .
\end{aligned}
$$


To compute $d_{r s}$ we use the matrix $\Delta^{R} \Delta M_{X}=\left(q_{r s}\right)$ :

$$
\begin{aligned}
q_{r s} & =c_{r s}-c_{r s-1} \\
& =1-\sum_{\substack{h \leq r \\
k \leq s}}\left(\alpha_{h k}-\beta_{h k}+\gamma_{h k}\right)-1+\sum_{\substack{h \leq r \\
k \leq s-1}}\left(\alpha_{h k}-\beta_{h k}+\gamma_{h k}\right) \\
& =-\sum_{h \leq r}\left(\alpha_{h s}-\beta_{h s}+\gamma_{h s}\right) ;
\end{aligned}
$$

now we can perform the last computation

$$
\begin{aligned}
d_{r s} & =q_{r s}-q_{r-1 s}=-\sum_{h \leq r}\left(\alpha_{h s}-\beta_{h s}+\gamma_{h s}\right)+\sum_{h \leq r-1}\left(\alpha_{h s}-\beta_{h s}+\gamma_{h s}\right) \\
& =-\alpha_{r s}+\beta_{r s}-\gamma_{r s} .
\end{aligned}
$$

(vii) Suppose that $(i, j) \geq(a+2, b+2)$ is the degree of a maximal first syzygy. Notice that $\alpha_{i j}=0$ by item (iv); moreover for $(i, j)>$ $(a+1, b+1)$ one has $d_{i j}=0$, and thus in the range $(r, s)>(i, j)$ we have $\alpha_{r s}=0$ and $\beta_{r s}=0$, which implies $\gamma_{r s}=0$ : so our syzygy is linked by no second syzygy. Hence, by item $(\mathrm{v}), m_{i-2 j-2}<\operatorname{deg} X$ must occur; this is a contradiction as $(i-2, j-2) \geq(a, b)$ and therefore $m_{i-2 j-2}=m_{a b}=\operatorname{deg} X$.

\section{Arithmetically Cohen-Macaulay 0-dimensional subschemes. As} we know not every 0 -dimensional subscheme $X \subset Q$ is ACM; in this section we want to characterize the ACM subschemes in term of their Hilbert matrix.

An admissible matrix $M^{\prime}$ will be called an $A C M$ matrix if $\Delta M^{\prime}$ has only nonnegative entries. If an ACM matrix $M^{\prime}$ of size $(a, b)$ is such that $\Delta M^{\prime}$ has entries $c_{i j}^{\prime}=1$ for every $(i, j) \leq(a, b)$, it is trivial to verify that $M^{\prime}$ is the Hilbert matrix of a complete intersection of type $(a+1,0),(0, b+1)$.

Let $M^{\prime}$ be an ACM matrix of size $(a, b)$. We say that $(i, j)$ is a corner for $\Delta M^{\prime}$ if $(i, j)=(0, b+1)$ or $(i, j)=(a+1,0)$, or even if $c_{i j}^{\prime}=0$ and $c_{i-1 j}^{\prime}=c_{i j-1}^{\prime}=1$. We say that $(i, j)$ is a vertex for $\Delta M^{\prime}$ if $c_{i-1 j}^{\prime}=c_{i j-1}^{\prime}=0$ and $c_{i-1 j-1}^{\prime}=1$; in this case, of course, $c_{i j}^{\prime}=0$. See Figure 1 . 


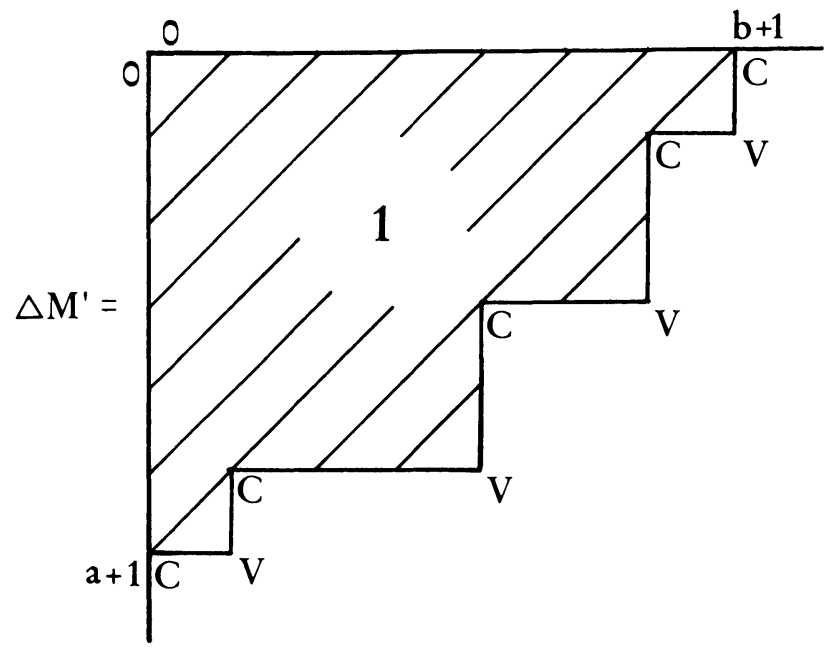

$\mathrm{C}=$ corner

FIGURE 1

One can check for an ACM matrix $M^{\prime}$ that the entries of $\Delta^{2} M^{\prime}=$ $\left(d_{i j}^{\prime}\right)$ are:

$$
d_{i j}^{\prime}= \begin{cases}1 & \text { if }(i, j)=(0,0) \text { or }(i, j) \text { is a vertex } \\ -1 & \text { if }(i, j) \text { is a corner } \\ 0 & \text { otherwise }\end{cases}
$$

Recall that $X \subset Q$ is an ACM 0-dimensional subscheme if and only if the minimal free resolution of $\mathscr{I}_{X}$ is of type (2) of $\S 3$ with $\gamma_{i j}=0$ for all $(i, j)$.

THEOREM 4.1. Let $X \subset Q$ be a 0-dimensional subscheme, and let $M_{X}$ be its Hilbert matrix. $X$ is an ACM scheme if and only if $M_{X}$ is an ACM matrix. Furthermore, in this case, the minimal free resolution of $\mathscr{I}_{X}$ looks like

$$
0 \rightarrow \bigoplus_{i=1}^{m-1} \mathscr{O}_{Q}\left(-a_{2 i},-a_{2 i}^{\prime}\right) \rightarrow \bigoplus_{i=1}^{m} \mathscr{O}_{Q}\left(-a_{1 i},-a_{1 i}^{\prime}\right) \rightarrow \mathscr{I}_{X} \rightarrow 0
$$

where $\left(a_{2 i}, a_{2 i}^{\prime}\right)$ runs over all the vertices and $\left(a_{1 i}, a_{1 i}^{\prime}\right)$ runs over all the corners of $\Delta M_{X}$.

Proof. For complete intersections the theorem is trivially true. Assume that $X$ is an ACM not c.i. subscheme. Suppose by contradiction that there are negative entries in $\Delta M_{X}=\left(c_{i j}\right)$ : take a maximal one, say $c_{r s}<0$ such that $c_{i j}=0$ for $(i, j)>(r, s)$. Such an element does exist by Proposition 2.7 and Remark 2.8. By the choice of $(r, s)$ 
one can write:

$$
d_{r+1 s+1}=c_{r+1 s+1}+c_{r s}-c_{r+1 s}-c_{r s+1}=c_{r s}<0 .
$$

Apply Proposition 3.3 item (vi): $d_{r+1 s+1}=-\alpha_{r+1 s+1}+\beta_{r+1 s+1}<0$ (recall that $\gamma_{i j}=0$ for all $\left.(i, j)\right)$; so, $\alpha_{r+1 s+1}>\beta_{r+1 s+1} \geq 0$ i.e. there is at least one minimal generator in degree $(r+1, s+1)$. This provides a contradiction since $d_{i j}=0$ for every $(i, j)>(r+1, s+1)$ while a syzygy is required by item (iv) of Proposition 3.3.

Vice versa, let us suppose that $M_{X}$ is an ACM matrix of size $(a, b)$. Applying Theorem 2.12 to $M_{X}$, one shows that there are $a+1(1,0)$ lines, $L_{i}(i=0,1, \ldots, a)$ each containing as many points of $X$ as the positive entries of the $i$ th row of $\Delta M_{X}$, and $b+1(0,1)$-lines, $L_{j}^{\prime} \quad(j=0,1, \ldots, b)$ each containing as many points of $X$ as the positive entries of the $j$ th column of $\Delta M_{X}$.

Claim 1. If $i \leq a$ or $j \leq b$, then

$$
\alpha_{i j}= \begin{cases}1 & \text { if }(1, j) \text { is a corner of } \Delta M_{X}, \\ 0 & \text { otherwise }\end{cases}
$$

To prove the claim we start with observing that if $(i, j)$ is a corner of $\Delta M_{X}$, then $h^{0}\left(\mathscr{I}_{X}(i, j)\right)=1$; hence $\alpha_{i j}=1$. Moreover, this generator is the curve of type $(i, j)$ consisting of the lines $L_{0}, L_{1}, \ldots$, $L_{i-1}$ and $L_{0}^{\prime}, L_{1}^{\prime}, \ldots, L_{j-1}^{\prime}$. Let us show, now, that for any other $(i, j)$ in our range, a curve of type $(i, j)$ containing $X$ is a combination of the previous generators. We suppose $i \leq a$ and work by induction on $b$ (a similar proof can be done when $j \leq b$ working by induction on $a$ ). When $b=0 \quad X \subset L_{0}^{\prime}$ is a c.i.; assume the statement true when $X$ is contained in less than $b+1(0,1)$-lines. In this case any curve $C$ of type $(i, j)$ through $X$ splits into $L^{\prime}$ and $C^{\prime}$, where $L^{\prime}$ is the union of the $r>0(0,1)$-lines containing more than $i$ points of $X$ and $C^{\prime}$ is a curve of type $(i, j-r)$ containing $Z=X-\left\{L^{\prime} \cap X\right\}$. By Corollary 2.16 the matrix $\Delta M_{Z}$ can be obtained from $\Delta M_{X}$ just deleting the columns $0,1, \ldots, r-1$; then every corner of $\Delta M_{Z}$ corresponds to a corner of $\Delta M_{X}$. By the inductive assumption $C^{\prime}$ is a combination of the generators of $I(Z)$ corresponding to the corners of $\Delta M_{Z}$. Now the multiplication by $L^{\prime}$ supplies the required expression for $C$.

If $(i, j)$ is a vertex, counting the dimension of $H^{0}\left(\mathscr{I}_{X}(i, j)\right)$ and taking into account that in each rectangle with opposite vertices $(0,0)$ 
and $(i, j)$ there are just two generators of $I(X)$, one shows that $\beta_{i j}=$ 1 .

Claim 2. If $\Sigma$ is a first syzygy which acts only on the generators corresponding to the corners, then it is generated by the syzygies on the vertices.

Let $\Sigma$ be such a syzygy. For simplicity, we restrict ourselves to the case when $\Delta M_{X}$ has three corners $(0, b+1),(r+1, s+1),(a+1,0)$; the procedure easily extends to the general case. In this hypothesis the three generators will be (recall that we do not distinguish between curves and the forms defining them):

$F_{1}=R \cdot R^{\prime} \quad$ where $R=L_{0}^{\prime} \cdot L_{1}^{\prime} \cdots \cdot L_{s}^{\prime}$ and $R^{\prime}=L_{s+1}^{\prime} \cdot L_{s+2}^{\prime} \cdots \cdot L_{b}^{\prime}$;

$F_{2}=R \cdot T \quad$ where $T=L_{1} \cdot L_{2} \cdots L_{r}$;

$F_{3}=T \cdot T^{\prime} \quad$ where $T^{\prime}=L_{r+1} \cdot L_{r+2} \cdots L_{a}$;

and the syzygies corresponding to the vertices will be:

$$
\begin{array}{ll}
\Sigma_{1}=\left(T,-R^{\prime}, 0\right) & \text { which links } F_{1} \text { and } F_{2}, \\
\Sigma_{2}=\left(0, T^{\prime},-R\right) & \text { which links } F_{2} \text { and } F_{3} .
\end{array}
$$

By the assumption, $\Sigma$ acts only on $F_{1}, F_{2}$, and $F_{3}$, so $\Sigma=$ $(X, Y, Z)$ with $X F_{1}+Y F_{2}+Z F_{3}=0$, i.e. $X F_{1}=-T\left(Y R+Z T^{\prime}\right)$. Since every $L_{i}$ in $T$ is not in $F_{1}$, it follows that $X=T X^{\prime}$; from which we get $X^{\prime} F_{1}+Y R+Z T^{\prime}=0$, i.e. $R\left(X^{\prime} R^{\prime}+Y\right)=-Z T^{\prime}$ and, with the same argument, we have $Z=R Z^{\prime}$. So, finally, we have $Y=-X^{\prime} R^{\prime}-Z^{\prime} T^{\prime}$. This implies:

$$
\Sigma=(X, Y, Z)=\left(T X^{\prime},-R^{\prime} X^{\prime}-Z^{\prime} T^{\prime}, R Z^{\prime}\right)=X^{\prime} \Sigma_{1}-Z^{\prime} \Sigma_{2} .
$$

Claim 3. If $i \leq a+1$ or $j \leq b+1$, then

$$
\beta_{i j}= \begin{cases}1 & \text { if }(i, j) \text { is a vertex of } \Delta M_{X}, \\ 0 & \text { otherwise }\end{cases}
$$

If $i \leq a$ or $j \leq b$, just apply Claim 2. If $i=a+1$ and $j \geq$ $b+1$ (resp. $j=b+1$ and $i \geq a+1$ ) we have $d_{a+1 j}=0$ (resp. $\left.d_{i b+1}=0\right)$. If for some $j \quad \beta_{a+1 j} \neq 0$, we could take the minimal $j$ with this property; a syzygy in this degree would have to act only on the generators of the corners: by Claim 2 this means $\beta_{a+1 j}=0$. The same argument works for $\beta_{i b+1}$. 
Conclusion. Recalling that $d_{i j}=-\alpha_{i j}+\beta_{i j}-\gamma_{i j}$, a simple computation shows that $\gamma_{i j}=0$ in the range $i \leq a+1$ or $j \leq b+1$; so, in the same range, $\alpha_{i j}=0$ outside the corners. On the other hand, for $(i, j) \geq(a+2, b+2)$ Proposition 3.3 item (vii) states $\alpha_{i j}=\beta_{i j}=\gamma_{i j}=0$ and the proof is complete.

Note that the Hilbert matrix of an ACM 0-dimensional subscheme of $Q$ completely determines the graded Betti numbers of its ideal sheaf, although this is not true for 0-dimensional subschemes of $\mathbf{P}^{n}$.

As we saw in Example 2.14 not every admissible matrix is the Hilbert matrix of some 0-dimensional subscheme of $Q$. We want to show that this happens for ACM matrices.

Theorem 4.2. Let $M^{\prime}=\left(m_{i j}^{\prime}\right)$ be an ACM matrix of size $(a, b)$. For any choice of $a+1$ distinct $(1,0)$-lines and $b+1(0,1)$-lines, there exists in their complete intersection one and only one (up to permutations of lines) subscheme $X$ such that $M^{\prime}=M_{X}$. Further $X$ is an ACM subscheme.

Proof. We construct a subscheme $X$ with the required property. Let $L_{0}, L_{1}, \ldots, L_{a}$ be any $a+1(1,0)$-lines, and $L_{0}^{\prime}, L_{1}^{\prime}, \ldots, L_{b}^{\prime}$ be any $b+1(0,1)$-lines. Set $P_{i j}=L_{i} \cap L_{j}^{\prime}(i=0,1, \ldots, a ; j=$ $0,1, \ldots, b)$ and consider $X=\left\{P_{i j} \mid c_{i j}^{\prime}=1\right\}$, where $\Delta M^{\prime}=\left(c_{i j}^{\prime}\right)$. We want to check that $M^{\prime}=M_{X}$. Of course, it is enough to verify that $m_{i j}=m_{i j}^{\prime}$ for $(i, j) \leq(a, b)$, since by definition of $X \Delta M_{X}(i, j)=$ $c_{i j}=c_{i j}^{\prime}=0$ for $i>a$ or $j>b$.

Note that, for $(i, j) \leq(a, b)$,

$$
m_{i j}^{\prime}=\sum_{r \leq i, s \leq j} c_{r s}^{\prime}=\#\left\{P_{r s} \in X \mid(r, s) \leq(i, j)\right\} .
$$

We have just to prove that $X$ gives $m_{i j}^{\prime}$ conditions to $H^{0}(i, j)$.

We work by induction on the number $a+1$ of $(1,0)$-lines containing $X$. If $a=0$ then $X$ consists of $b+1$ collinear points; so, $m_{0 j}=\min \{j+1, b+1\}=m_{0 j}^{\prime}$ for every $j$.

Inductive step. By construction, $L_{0}$ contains $b+1$ points of $X$; hence every curve $C$ of type $(i, j)$ through $X$ must contain it since $j<b+1$. Thus, $C=L_{0} \cdot C^{\prime}$, where $C^{\prime}$ is a curve of type $(i-1, j)$ containing $\bar{X}=X-\left\{P_{00}, P_{01}, \ldots, P_{0 b}\right\}$. Let $\Delta \bar{M}=\left(\bar{c}_{i j}\right)$ be the matrix obtained from $\Delta M^{\prime}$ by deleting the first row; we have $\bar{c}_{i j}=$ $c_{i+1 j}^{\prime}$ for $i \geq 0 \quad\left(\bar{c}_{i j}=0\right.$ for $\left.i<0\right)$. Notice that $\bar{X}$ is the set of points which one can construct from $\bar{M}=\left(\bar{m}_{i j}\right)$ with the same procedure 
we did for $X$ from $M^{\prime} . \Delta \bar{M}$ has " $a$ " rows; so we have

$$
m_{i j}=M_{\bar{X}}(i-1, j)+j+1=\bar{m}_{i-1 j}+j+1=m_{i j}^{\prime}
$$

where the first equality comes from the definition of $X$, the second from the inductive hypothesis and the third by a straight computation.

We prove uniqueness again by induction on $a+1$.

If $a=0$ then $X$ is the complete intersection $L_{0} \cap\left(\bigcup_{j=0}^{b} L_{j}^{\prime}\right)$. Let $Y$ be another subscheme of the c.i. $\left(\bigcup_{i=0}^{a} L_{i}\right) \cap\left(\bigcup_{j=0}^{b} L_{j}^{\prime}\right)$ such that $M_{Y}=M^{\prime}$ and let again $L_{0}$ be one of the $(1,0)$-lines containing $b+1$ points of $Y$. By the inductive assumption one has:

$$
Y-\left\{Y \cap L_{0}\right\}=\bar{X}
$$

therefore $Y=X$. The last claim is Theorem 4.1 .

REMARK 4.3. We already know that there are 0-dimensional subschemes $X$ of $Q$ which are ideally c.i. but not c.i. (see Remark 3.2). In the case of ACM subschemes we have: $X$ is ideally c.i. if and only if $X$ is c.i. In fact, if $X \subset Q$ is an ACM 0-dimensional subscheme which is not c.i., then a minimal set of generators for the ideal $I(X)$ is given in Theorem 4.1: the two generators of degree $(a+1,0)$, $(0, b+1)$ defines a c.i.; any other pair of generators has a common component (which is a union of lines). So, $X$ cannot be ideally c.i.

REMARK 4.4. Let $\bar{H}$ be the following sequence of integers, and $\Delta \bar{H}$ its first difference

$$
\bar{H}: 1,4,9, \ldots, b^{2}, b^{2}+c_{1}, b^{2}+c_{1}+c_{2}, \ldots, b^{2}+\sum_{i=1}^{t} c_{i}, \rightarrow
$$

$\Delta \bar{H}: 1,3,5, \ldots, 2 b-1, c_{1}, c_{2}, \ldots, c_{t}, 0, \rightarrow$

(" $\rightarrow$ " means that the sequence stabilizes) where $2 b \geq c_{i} \geq c_{i+1}, i=$ $1,2, \ldots, t-1$. In [R2] was proved that there exists a subscheme $X \subset$ $\mathbf{P}^{3}$ on an irreducible quadric such that $H F(X)=\bar{H}$. Now we can construct a class of ACM matrices $M=\left(m_{i j}\right)$ such that $\bar{H}=\left\{m_{i i}\right\}$ : this will imply, by Theorem 4.2, that there are ACM 0-dimensional subschemes on a quadric $Q$ having $\bar{H}$ as their Hilbert function.

To construct $\Delta M$, we start with an ACM matrix $B$ of size $(b-1$, $b-1)$ whose entries are all " 1 "'s. Choose then $t$ couples $\left(p_{i}, q_{i}\right)$ such that $p_{i}+q_{i}=c_{i}$ and $b \geq p_{i} \geq p_{i+1}, b \geq q_{i} \geq q_{i+1}$ (this can be done by the assumption $2 b \geq c_{i} \geq c_{i+1}$ ). Now we border $B$ by $t$ rows (resp. $t$ columns) containing in the initial $p_{i}$ places (resp. in the 
initial $q_{i}$ places) " 1 " entries, and " 0 " elsewhere. The ACM matrix so obtained has the required properties.

REMARK 4.5. Let $X \subset Q$ be an ACM 0-dimensional subscheme and $M_{X}$ its Hilbert matrix, say of size $(a, b)$. Recall that the resolution of $\mathscr{I}_{X}$ is of the kind

$$
0 \rightarrow \bigoplus_{i=1}^{m-1} \mathscr{O}_{Q}\left(-a_{2 i},-a_{2 i}^{\prime}\right) \rightarrow \bigoplus_{i=1}^{m} \mathscr{O}_{Q}\left(-a_{1 i},-a_{1 i}^{\prime}\right) \rightarrow \mathscr{I}_{X} \rightarrow 0
$$

Applying the results of [PS] to our case, i.e. to the ring $S$ localized at its maximal irrelevant ideal $(\mathfrak{u}, \mathfrak{v})$, one has the following facts:

(i) $X$ is ACM if and only if the subscheme $X^{\prime}$ directly linked to $X$ in a c.i. is again ACM.

(ii) $X$ is ACM if and only if it is linked to a complete intersection; more precisely, if $m=\nu(I(X))$ is the number of elements in any minimal set of generators of $I(X)$, then $m-2$ is the minimal number of direct linkages

$$
X \sim X_{1} \sim \cdots \sim X_{m-1}
$$

in order that $X_{m-1}$ be a complete intersection.

(iii) We know that in any minimal set of generators of $I(X)$ there is a unique regular sequence consisting of two elements $f, g$ of type $(a+1,0),(0, b+1)$. One can use Ferrand's procedure, as shown in [PS], to find the resolution of $X^{\prime}$, the subscheme directly linked to $X$ in the c.i. $f, g$ :

$$
\begin{aligned}
0 & \rightarrow \bigoplus_{i=1}^{m-2} \mathscr{O}_{Q}\left(a_{1 i}-a-1, a_{1 i}^{\prime}-b-1\right) \\
& \rightarrow \bigoplus_{i=1}^{m-1} \mathscr{O}_{Q}\left(a_{2 i}-a-1, a_{2 i}^{\prime}-b-1\right) \rightarrow \mathscr{I}_{X^{\prime}} \rightarrow 0
\end{aligned}
$$

Moreover, if $M_{X^{\prime}}$ is the Hilbert matrix of $X^{\prime}$, setting $\Delta M_{X}=\left(c_{i j}\right)$ and $\Delta M_{X^{\prime}}=\left(c_{i j}^{\prime}\right)$ we have:

$$
c_{i j}^{\prime}= \begin{cases}1 & \text { if } c_{a-i b-j}=0 \text { with }(i, j) \leq(a, b) \\ 0 & \text { otherwise }\end{cases}
$$

Alternatively, one can say that for $(i, j) \leq(a, b) c_{i j}+c_{i j}^{\prime}=1$. One can easily realize how $\Delta M_{X^{\prime}}$ looks like, just giving a glance at Figure 2. 

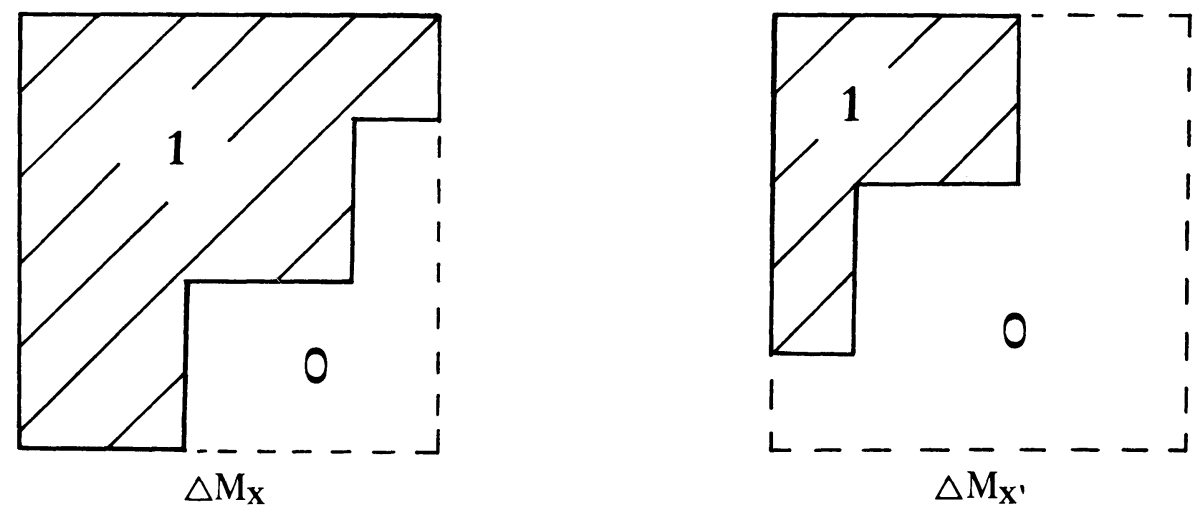

\section{FIGURE 2}

\section{REFERENCES}

[AF] F. W. Anderson and K. R. Fuller, Rings and Categories of Modules, GTM 13, Springer-Verlag, New York, 1973.

[B] E. Ballico, Generators for the homogeneous ideal of $s$ general points in $\mathbf{P}^{3}$, J. Algebra, 106 (1987), 46-52.

[BG] E. Ballico and A. V. Geramita, The minimal free resolution of the ideal of $s$ general points in $\mathbf{P}^{3}$, Canad. Math. Soc. Conf. Proc., 6 (1986), 1-10.

[CGO] C. Ciliberto, A. V. Geramita, and F. Orecchia, Perfect varieties with defining equations of high degree, Boll. Un. Mat. Ital. 7, 1-B, (1987), 633-647.

[D] E. Davis, 0-dimensional subschemes of $\mathbf{P}^{2}$ : new application of Castelnuovo function, Ann. Univ. Ferrara, sez. VII, Sc. Mat., 32 (1986), 93-107.

[DGO] E. Davis, A. V. Geramita, and F. Orecchia, Gorenstein algebras and the Cayley-Bacharach theorem, Proc. Amer. Math. Soc., 93 (1985), 593-597.

[E] G. Ellingsrud, Sur le schéma de Hilbert des variétés de codimension 2 dans $\mathbf{P}^{e}$ à cône de Cohen-Macaulay, Ann. Sc. Ec. Norm. Sup., t. 8 fasc. 4 (1975), 423-431.

[GM] A. V. Geramita and P. Maroscia, The ideal of forms vanishing at a finite set of points in $\mathbf{P}^{n}$, J. Algebra, 90 (1984), 528-555.

[GMR] A. V. Geramita, P. Maroscia, and L. Roberts, The Hilbert function of a reduced k-algebra, J. London Math. Soc., (2), 28 (1983), 443-452.

[G] S. Giuffrida, Hilbert function of a 0-cycle in $\mathbf{P}^{2}$, Le Matematiche, Vol. XV, Fasc. I-II (1985), 252-266.

[GMa] S. Giuffrida and R. Maggioni, On the Rao module of a curve lying on a smooth cubic surface in $\mathbf{P}^{3}$, Comm. in Algebra, 18 (7), (1990), 2039-2061.

[GP1] L. Gruson and C. Peskine, Genre des courbes de l'espace projectif, Algebraic Geometry, Lecture Notes in Math. no. 687, Springer, 1978.

[GP2] _-, Section plane d'une courbe gauche: postulation, Prog. in Math., 24 Birkhauser (1982), 33-35.

[Hb] B. Harbourne, The geometry of rational surfaces and Hilbert functions of points in the plane, Canad. Math. Soc., Conf. Proc., 6 (1986), 95-111.

[HE] J. Harris and D. Eisenbud, Curves in projective space, Sem. de Math. Super. Université de Montréal, 1982.

[H] R. Hartshorne, Algebraic Geometry, GTM 52, Springer-Verlag, Berlin, 1977. 
[MR1] R. Maggioni and A. Ragusa, Connections between Hilbert function and geometric properties for a finite set of points in $\mathbf{P}^{2}$, Le Matematiche, Vol. XXXIX, Fasc. I-II (1984), 153-170.

[MR2] - The Hilbert function of generic plane sections of curves in $\mathbf{P}^{3}$, Inv. Math., 91 (1988), 253-258.

[M] P. Maroscia, Some problems and results on finite sets of points in $\mathbf{P}^{2}$, Lecture Notes in Math. no. 977, Springer-Verlag (1982), 290-314.

[MV] P. Maroscia and W. Vogel, On the defining equations of points in general position in $\mathbf{P}^{n}$, Math. Ann., 269 (1984), 183-189.

[PS] C. Peskine and L. Szpiro, Liaison des variétés algébriques I, Invent. Math., 26 (1974), 271-302.

[R1] G. Raditi, Hilbert function and geometric properties for a closed zero-dimensional subscheme of a quadric $Q \subset \mathbf{P}^{3}$, to appear on Comm. in Algebra.

[R2] - Construction of a set of points on a smooth quadric $Q \subset \mathbf{P}^{3}$ with assigned Hilbert function, Queen's Papers in Pure and Applied Math., 83 (1989), art. J.

[Sa] T. Sauer, The number of equations defining points in general position, Pacific J. Math., 120 (1985), 199-213.

[St] R. Stanley, Hilbert function of graded algebras, Adv. in Math., 28 (1978), 57-83.

[S] R. Strano, Sulle sezioni iperpiane delle curve, Rend. Sem. Mat. e Fis. Milano, 57 (1987), 125-134.

Received August 14, 1990. Work was done with financial support of M.P.I., while the authors were members of C.N.R.

Dipartimento di Matematica

VIALE A. DoRIA, 6

95125 CATANia, ItALY 


\section{PACIFIC JOURNAL OF MATHEMATICS EDITORS}

\author{
V. S. VARADARAJAN \\ (Managing Editor) \\ University of California \\ Los Angeles, CA 90024-1555 \\ vsv@math.ucla.edu \\ Herbert Clemens \\ University of Utah \\ Salt Lake City, UT 84112 \\ clemens@math.utah.edu \\ F. Michael Christ \\ University of California \\ Los Angeles, CA 90024-1555 \\ christ@math.ucla.edu \\ THOMAs ENRIGHT \\ University of California, San Diego \\ La Jolla, CA 92093 \\ tenright@ucsd.edu
}

\author{
Nicholas ERCOLANI \\ University of Arizona \\ Tucson, AZ 85721 \\ ercolani@math.arizona.edu \\ R. FINN \\ Stanford University \\ Stanford, CA 94305 \\ finn@gauss.stanford.edu \\ VAUGHAN F. R. Jones \\ University of California \\ Berkeley, CA 94720 \\ vfr@math.berkeley.edu \\ Steven KerckhofF \\ Stanford University \\ Stanford, CA 94305 \\ spk@gauss.stanford.edu
}

\author{
C. C. MOORE \\ University of California \\ Berkeley, CA 94720
}

Martin ScharlemanN

University of California

Santa Barbara, CA 93106

mgscharl@henri.ucsb.edu

Harold Stark

University of California, San Diego La Jolla, CA 92093

\section{R. ARENS \\ ASSOCIATE EDITORS}

\begin{tabular}{lccc} 
R. ARENS & $\begin{array}{c}\text { E. F. BECKENBACH } \\
(1906-1982)\end{array}$ & B. H. NEUMANN & \multicolumn{1}{c}{ F. WOLF } \\
$(1904-1989)$ & K. YoshIDA \\
SUPPORTING & INSTITUTIONS \\
UNIVERSITY OF ARIZONA & UNIVERSITY OF OREGON \\
UNIVERSITY OF BRITISH COLUMBIA & UNIVERSITY OF SOUTHERN CALIFORNIA \\
CALIFORNIA INSTITUTE OF TECHNOLOGY & STANFORD UNIVERSITY \\
UNIVERSITY OF CALIFORNIA & UNIVERSITY OF HAWAII \\
MONTANA STATE UNIVERSITY & UNIVERSITY OF TOKYO \\
UNIVERSITY OF NEVADA, RENO & UNIVERSITY OF UTAH \\
NEW MEXICO STATE UNIVERSITY & WASHINGTON STATE UNIVERSITY \\
OREGON STATE UNIVERSITY & UNIVERSITY OF WASHINGTON \\
\hline
\end{tabular}

The Supporting Institutions listed above contribute to the cost of publication of this Journal, but they are not owners or publishers and have no responsibility for its content or policies.

Mathematical papers intended for publication in the Pacific Journal of Mathematics should be in typed form or offset-reproduced (not dittoed), double spaced with large margins. Please do not use built up fractions in the text of the manuscript. However, you may use them in the displayed equations. Underline Greek letters in red, German in green, and script in blue. The first paragraph must be capable of being used separately as a synopsis of the entire paper. In particular it should contain no bibliographic references. Please propose a heading for the odd numbered pages of less than 35 characters. Manuscripts, in triplicate, may be sent to any one of the editors. Please classify according to the 1991 Mathematics Subject Classification scheme which can be found in the December index volumes of Mathematical Reviews. Supply name and address of author to whom proofs should be sent. All other communications should be addressed to the managing editor, or Elaine Barth, University of California, Los Angeles, California 90024-1555.

There are page-charges associated with articles appearing in the Pacific Journal of Mathematics. These charges are expected to be paid by the author's University, Government Agency or Company. If the author or authors do not have access to such Institutional support these charges are waived. Single authors will receive 50 free reprints; joint authors will receive a total of 100 free reprints. Additional copies may be obtained at cost in multiples of 50 .

The Pacific Journal of Mathematics (ISSN 0030-8730) is published monthly except for July and August. Regular subscription rate: $\$ 190.00$ a year (10 issues). Special rate: $\$ 95.00$ a year to individual members of supporting institutions.

Subscriptions, orders for numbers issued in the last three calendar years, and changes of address should be sent to Pacific Journal of Mathematics, P.O. Box 969, Carmel Valley, CA 93924, U.S.A. Old back numbers obtainable from Kraus Periodicals Co., Route 100, Millwood, NY 10546.

The Pacific Journal of Mathematics at P.O. Box 969, Carmel Valley, CA 93924 (ISSN 0030-8730) is published monthly except for July and August. Second-class postage paid at Carmel Valley, California 93924, and additional mailing offices. Postmaster: send address changes to Pacific Journal of Mathematics, P.O. Box 969, Carmel Valley, CA 93924.

PUBLISHED BY PACIFIC JOURNAL OF MATHEMATICS, A NON-PROFIT CORPORATION Copyright (C) 1992 by Pacific Journal of Mathematics 


\section{PACIFIC JOURNAL OF MATHEMATICS}

Volume $155 \quad$ No. $2 \quad$ October 1992

Invariant subspaces and harmonic conjugation on compact abelian

201 groups

NAKHLE HABIB ASMAR, EARL ROBERT BERKSON and

ThOMAS Alastair GiLlESPIE

Cobcat and singular bordism

A. K. DAS and S. S. KHARE

Poles of Eisenstein series on $\mathrm{SL}_{n}$ induced from maximal parabolics

PAUL FEIT

On the postulation of 0-dimensional subschemes on a smooth quadric

251

SAlvatore GiUfFrida, Renato MagGioni and AlFio

RAGUSA

The Euler class for "piecewise" groups

Peter Abraham GREenberg

$L^{p}$-Fourier transforms on nilpotent Lie groups and solvable Lie groups 295 acting on Siegel domains

JUNKO INOUE

Fuchsian moduli on a Riemann surface-its Poisson structure and

Poincaré-Lefschetz duality

KATSUNORI IWASAKI

Interpolation between $H^{p}$ spaces and noncommutative generalizations. 341 I

\section{GILLES PISIER}

The braid index of generalized cables

ROBERT FONES WILLIAMS and ROBERT FONES WILLIAMS $B M O$ and Hankel operators on Bergman spaces 